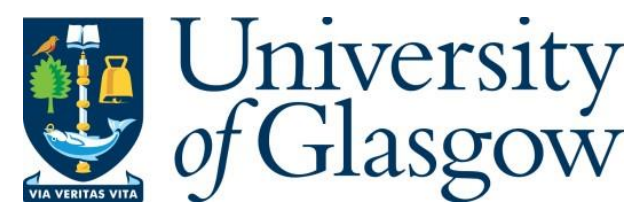

Lee, C. H., Gil, A. J., Hassan, O. I., Bonet, J. and Kulasegaram, S. (2017) A variationally consistent Streamline Upwind Petrov-Galerkin Smooth Particle Hydrodynamics algorithm for large strain solid dynamics. Computer Methods in Applied Mechanics and Engineering, 318, pp. 514-536.

There may be differences between this version and the published version. You are advised to consult the publisher's version if you wish to cite from it.

$\underline{\text { http://eprints.gla.ac.uk/168849/ }}$

Deposited on: 14 September 2018

Enlighten - Research publications by members of the University of Glasgow http://eprints.gla.ac.uk 


\section{Accepted Manuscript}

A variationally consistent Streamline Upwind Petrov Galerkin Smooth Particle Hydrodynamics algorithm for large strain solid dynamics

Chun Hean Lee, Antonio J. Gil, Osama I. Hassan, Javier Bonet, Sivakumar Kulasegaram

PII: S0045-7825(16)31520-1

DOI: $\quad$ http://dx.doi.org/10.1016/j.cma.2017.02.002

Reference: CMA 11329

To appear in: Comput. Methods Appl. Mech. Engrg.

Received date: 10 November 2016

Revised date: 30 January 2017

Accepted date: 2 February 2017

Please cite this article as: C.H. Lee, A.J. Gil, O.I. Hassan, J. Bonet, S. Kulasegaram, A variationally consistent Streamline Upwind Petrov Galerkin Smooth Particle Hydrodynamics algorithm for large strain solid dynamics, Comput. Methods Appl. Mech. Engrg. (2017), http://dx.doi.org/10.1016/j.cma.2017.02.002

This is a PDF file of an unedited manuscript that has been accepted for publication. As a service to our customers we are providing this early version of the manuscript. The manuscript will undergo copyediting, typesetting, and review of the resulting proof before it is published in its final form. Please note that during the production process errors may be discovered which could affect the content, and all legal disclaimers that apply to the journal pertain. 


\title{
A variationally consistent Streamline Upwind Petrov Galerkin Smooth Particle Hydrodynamics algorithm for large strain solid dynamics
}

\author{
Chun Hean Lee ${ }^{a, 1}$, Antonio J. Gil ${ }^{a, 2}$, Osama I. Hassan ${ }^{a}$, Javier Bonet ${ }^{b}$, Sivakumar \\ Kulasegaram $^{c}$ \\ (a) Zienkiewicz Centre for Computational Engineering, College of Engineering \\ Swansea University, Bay Campus, SA1 8EN, United Kingdom \\ (b) University of Greenwich, London, SE10 9LS, United Kingdom \\ (c) School of Engineering, Cardiff University, Cardiff CF24 3AA, United Kingdom
}

\begin{abstract}
This paper presents a new Smooth Particle Hydrodynamics (SPH) computational framework for explicit fast solid dynamics. The proposed methodology explores the use of the Streamline Upwind Petrov Galerkin (SUPG) stabilisation methodology as an alternative to the Jameson-Schmidt-Turkel (JST) stabilisation recently presented by the authors in [1] in the context of a conservation law formulation of fast solid dynamics. The work introduced in this paper puts forward three advantageous features over the recent JST-SPH framework. First, the variationally consistent nature of the SUPG stabilisation allows for the introduction of a locally preserving angular momentum procedure which can be solved in a monolithic manner in conjunction with the rest of the system equations. This differs from the JST-SPH framework, where an a posteriori projection procedure was required to ensure global angular momentum preservation. Second, evaluation of expensive harmonic and bi-harmonic operators, necessary for the JST stabilisation, is circumvented in the new SUPG-SPH framework. Third, the SUPG-SPH framework is more accurate (for the same number of degrees of freedom) than its JST-SPH counterpart and its accuracy is comparable to that of the robust (but computationally more demanding) Petrov Galerkin Finite Element Method (PG-FEM) technique explored by the authors in [2-5], as shown in the numerical examples included. A series of numerical examples are analysed in order to benchmark and assess the robustness and effectiveness of the proposed algorithm. The resulting SUPG-SPH framework is therefore accurate, robust and computationally efficient, three key desired features that will allow the authors in forthcoming publications to explore its applicability in large scale simulations.
\end{abstract}

Keywords: Conservation laws, SPH, Instability, SUPG, Fast dynamics, Incompressibility

\section{Introduction}

Traditionally, the displacement-based Smooth Particle Hydrodynamics (SPH) Lagrangian formalism [6-12] has suffered from a number of well-known shortcomings, namely the presence

\footnotetext{
${ }^{1}$ Corresponding author: c.h.lee@swansea.ac.uk

${ }^{2}$ Corresponding author: a.j.gil@swansea.ac.uk
} 
of numerical errors near boundaries due to a reduced compact support [13, 14]; the presence of tensile instability which results in a non-physical clumping of particles [15]; the appearance of hourglassing due to the rank-deficiency inherent to the use of under-integrated particle (or nodal) integration [16] and the reduced order of convergence for derived variables (i.e. stresses and strains) [17-20].

In order to circumvent the above shortcomings, significant effort has been devoted to enhance the robustness of (displacement-based) SPH algorithms. New sophisticated kernels and gradient corrections have been used in order to ensure reproducibility (consistency) of complete polynomial basis in finite domains [7, 21-23]. However, as reported in References $[8,10]$, the above enhanced SPH methodologies still suffer from persistent artificial mechanisms similar to hour-glassing when attempting to model problems with predominant nearly incompressible behaviour. These numerical deficiencies can be partially alleviated through the use of so-called non-consistent stabilisation strategies (i.e. artificial viscous fluxes $[6,24,25]$ and conservative strain smoothing regularisation $[17,26])$.

Some interesting work has been reported in [27-29], where a mixed formulation in conjunction with a Taylor-Galerkin stabilisation algorithm is employed for the simulation of a viscoplastic continuum. Unfortunately, the introduction of appropriate numerical viscosity (leading to a robust framework) through a variationally consistent stabilisation procedure is still not clear for the latter formulation. Furthermore, its physical interpretation is debatable, especially in the context of non-dissipative reversible processes.

In a very recent work [1], some of the authors of the present manuscript have successfully introduced a new SPH computational framework for explicit fast solid dynamics, where the conservation of linear momentum $\boldsymbol{p}$ is solved along with conservation equations for the deformation gradient $\boldsymbol{F}$, its co-factor $\boldsymbol{H}$ and its Jacobian $J$. Specifically, SPH discretisation of the new mixed system of conservation laws $\{\boldsymbol{p}, \boldsymbol{F}, \boldsymbol{H}, J\}[3,5]$ is introduced in conjunction with a well-established Jameson-Schmidt-Turkel (JST) [30] stabilisation methodology. The resulting JST-SPH framework was capable of eliminating spurious hourglass-like modes, tensile instability and spurious oscillations in nearly incompressible scenarios.

The main objective of the present manuscript is to further explore the SPH discretisation of the mixed based system in [1] by means of an alternative variationally consistent Streamline Upwind Petrov Galerkin (SUPG) [31] stabilisation methodology. In addition to a higher computational efficiency to that of the JST-SPH algorithm (e.g. harmoic and bi-harmonic dissipative operators are not required), the variationally consistent nature of the proposed SUPG-SPH framework allows for the conservation of linear and angular momenta without the need to introduce an a posteriori projection algorithm. Crucially, the computational efficiency and excellent performance of the proposed framework in nearly incompressible scenarios open up interesting possibilities in terms of its applicability in the field of biomechanics, typically accompanied with severe mesh distortions that the algorithm proposed (meshless-based) can efficiently handle.

The paper is organised as follows. Section 2 presents the complete mixed-based $\{\boldsymbol{p}, \boldsymbol{F}, \boldsymbol{H}, J\}$ set of first order conservation laws for solid dynamics. Sections 3 and 4 describe the computational methodology of the SUPG-SPH framework. The variational statement of the mixed-based $\{\boldsymbol{p}, \boldsymbol{F}, \boldsymbol{H}, J\}$ system, the SPH spatial discretisation and a variationally consistent SUPG algorithm are also presented. For clarity, the complete SUPG-SPH flowchart is summarised in Section 5. Section 6 describes a monolithic projection algorithm used to locally preserve angular momentum. Section 7 presents the algorithmic description of the 


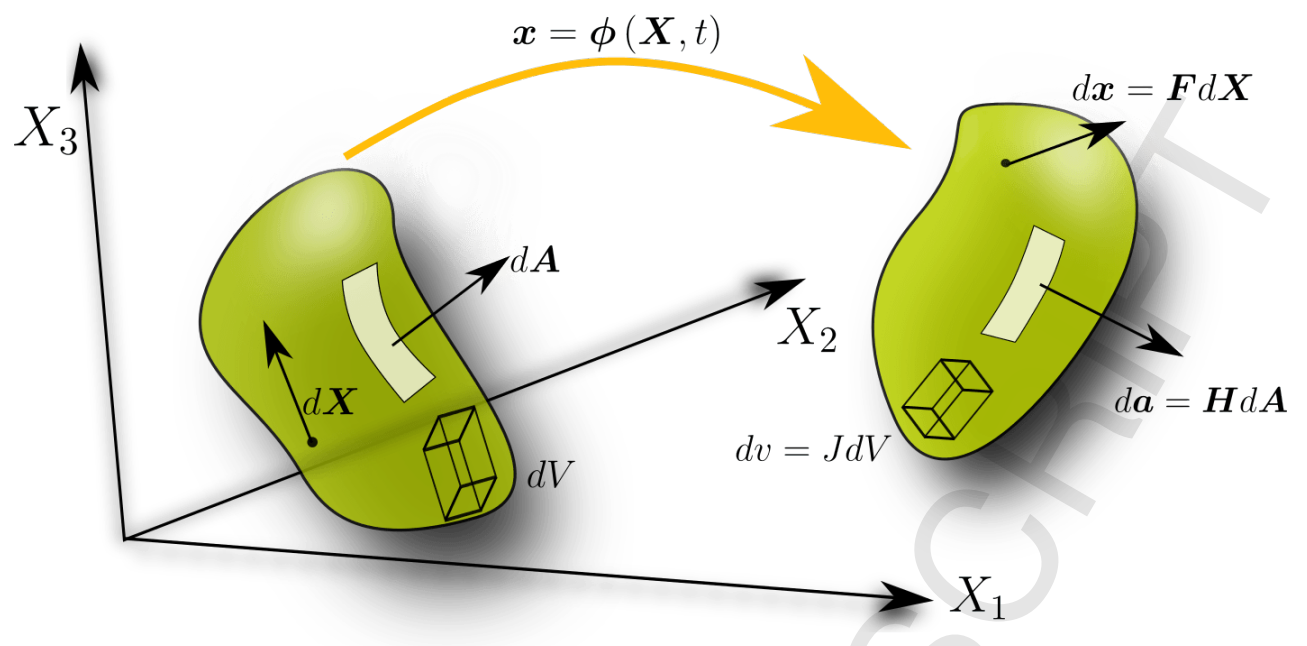

Figure 1: Motion of a continuum domain

proposed $\{\boldsymbol{p}, \boldsymbol{F}, \boldsymbol{H}, J\}$ SUPG-SPH methodology. In Section 8, an extensive set of challenging numerical examples is presented to assess the performance of the proposed methodology. Finally, Section 9 presents some concluding remarks and current directions of research.

\section{Reversible elastodynamics}

Consider the three dimensional deformation of an elastic body of material density $\rho_{0}$ moving from its undeformed configuration occupying a volume $V$, of boundary $\partial V$, to a deformed configuration at time $t$ occupying a volume $v$, of boundary $\partial v$ (see Figure 1 ). The motion is defined through a deformation mapping $\boldsymbol{x}=\boldsymbol{\phi}(\boldsymbol{X}, t)$ which satisfies the following mixed set of Total Lagrangian conservation laws [1, 2, 32-36]:

$$
\begin{aligned}
& \frac{\partial \boldsymbol{p}}{\partial t}-\mathrm{DIV} \boldsymbol{P}=\boldsymbol{f}_{0} \\
& \frac{\partial \boldsymbol{F}}{\partial t}-\nabla_{0} \boldsymbol{v}=\mathbf{0} \\
& \frac{\partial \boldsymbol{H}}{\partial t}-\operatorname{CURL}(\boldsymbol{v} \times \boldsymbol{F})=\mathbf{0} \\
& \frac{\partial J}{\partial t}-\operatorname{DIV}\left(\boldsymbol{H}^{T} \boldsymbol{v}\right)=0
\end{aligned}
$$

where $\boldsymbol{v}$ is the velocity field (with $\boldsymbol{p}:=\rho_{0} \boldsymbol{v}$ the linear momentum per unit of undeformed volume), $\boldsymbol{F}$ is the deformation gradient (or fibre map), $\boldsymbol{H}$ is the co-factor of the deformation (or area map), $J$ is the Jacobian of the deformation (or volume map), $\boldsymbol{P}$ is the first PiolaKirchhoff stress tensor and $\boldsymbol{f}_{0}$ is a body force term per unit of undeformed volume. The symbol $\times$ represents the tensor cross product between vectors and/or second order tensors in the sense of $[4,5,37,38]$, DIV and CURL represent the material divergence and curl operators as defined in (5) and (7) of Reference [5], respectively, and $\boldsymbol{\nabla}_{0}$ represents the material gradient operator defined as $\boldsymbol{\nabla}_{0}:=\frac{\partial}{\partial \boldsymbol{X}}$. 
Above equation (1a) represents the conservation of linear momentum whilst equations (1b)-(1d) represent a set of compatibility equations for the strain measures $\{\boldsymbol{F}, \boldsymbol{H}, J\}$. As already shown in $[1,3]$, appropriate geometric involutions must be satisfied by the strain measures $\boldsymbol{F}$ and $\boldsymbol{H}$ [39] as

$$
\mathrm{CURL} \boldsymbol{F}=\mathbf{0} ; \quad \text { DIV } \boldsymbol{H}=\mathbf{0} .
$$

Notice that, if necessary, the use of these involutions enables the conservation equations for the area and volume maps in (1c) and (1d), respectively, to be re-written as [1]

$$
\frac{\partial \boldsymbol{H}}{\partial t}-\boldsymbol{F} \times \nabla_{0}\left(\frac{\boldsymbol{p}}{\rho_{0}}\right)=\mathbf{0} ; \quad \frac{\partial J}{\partial t}-\boldsymbol{H}: \nabla_{0}\left(\frac{\boldsymbol{p}}{\rho_{0}}\right)=0
$$

The above system of conservation laws (1) can alternatively be summarised in a concise manner as

$$
\frac{\partial \mathcal{U}}{\partial t}+\frac{\partial \mathcal{F}_{I}}{\partial X_{I}}=\mathcal{S} ; \quad \forall I=1,2,3
$$

where $\mathcal{U}$ denotes the set of conservation variables, $\mathcal{S}$ the source term and $\mathcal{F}_{I}$ the flux vector in the Cartesian direction $I$, as follows

$$
\mathcal{U}=\left[\begin{array}{c}
\boldsymbol{p} \\
\boldsymbol{F} \\
\boldsymbol{H} \\
J
\end{array}\right] ; \quad \mathcal{F}_{I}=-\left[\begin{array}{c}
\boldsymbol{P} \boldsymbol{E}_{I} \\
\frac{1}{\rho_{0}} \boldsymbol{p} \otimes \boldsymbol{E}_{I} \\
\boldsymbol{F} \times\left(\frac{1}{\rho_{0}} \boldsymbol{p} \otimes \boldsymbol{E}_{I}\right) \\
\boldsymbol{H}:\left(\frac{1}{\rho_{0}} \boldsymbol{p} \otimes \boldsymbol{E}_{I}\right)
\end{array}\right] ; \quad \mathcal{S}=\left[\begin{array}{c}
\boldsymbol{f}_{0} \\
\mathbf{0} \\
\mathbf{0} \\
0
\end{array}\right]
$$

with $\boldsymbol{E}_{I}$ is the $I$-th unit vector of the Cartesian basis defined as

$$
\boldsymbol{E}_{1}=\left[\begin{array}{c}
1 \\
0 \\
0
\end{array}\right] ; \quad \boldsymbol{E}_{2}=\left[\begin{array}{l}
0 \\
1 \\
0
\end{array}\right] ; \quad \boldsymbol{E}_{3}=\left[\begin{array}{l}
0 \\
0 \\
1
\end{array}\right]
$$

For the particular case of a reversible process, the closure of the system of conservation laws in (1) (or (4)) requires the introduction of an appropriate constitutive law relating the stress tensor $\boldsymbol{P}$ and the strain measures $\{\boldsymbol{F}, \boldsymbol{H}, J\}$. Finally, for the complete definition of the Initial Boundary Value Problem (IBVP), initial and boundary (essential and natural) conditions must also be specified as appropriate.

\subsection{Constitutive model: nearly incompressible polyconvexity}

In this work, and without loss of generality, a hyperelastic polyconvex nearly incompressible constitutive model has been considered $^{3}$, where the strain energy density is defined as a convex multi-variable function $W$ of the strain measures $\{\boldsymbol{F}, \boldsymbol{H}, J\}$ as

$$
W=\hat{W}+U ; \quad \hat{W}=\varsigma J^{-2 / 3}\left(I_{1}\right)+\xi J^{-2}\left(I_{2}\right)^{3 / 2} ; \quad U=\frac{\kappa}{2}(J-1)^{2},
$$

\footnotetext{
${ }^{3}$ A detailed discussion of this model can be found in Section 2.1 of Reference [1] on pg. 75 .
} 
where $I_{1}=\boldsymbol{F}: \boldsymbol{F}, I_{2}=\boldsymbol{H}: \boldsymbol{H}$ and $\varsigma, \xi$ and $\kappa$ (bulk modulus) are positive material parameters. The so-called conjugate stresses $\left\{\boldsymbol{\Sigma}_{\boldsymbol{F}}, \boldsymbol{\Sigma}_{\boldsymbol{H}}, \Sigma_{J}\right\}$ [4] (see Section 3 on pg. 149) are defined by

$$
\begin{aligned}
\boldsymbol{\Sigma}_{\boldsymbol{F}} & :=\frac{\partial W}{\partial \boldsymbol{F}}=2 \varsigma J^{-2 / 3} \boldsymbol{F} ; \\
\boldsymbol{\Sigma}_{\boldsymbol{H}} & :=\frac{\partial W}{\partial \boldsymbol{H}}=3 \xi J^{-2}\left(I_{2}\right)^{1 / 2} \boldsymbol{H} ; \\
\Sigma_{J} & :=\frac{\partial W}{\partial J}=-\frac{2 \varsigma}{3} J^{-5 / 3}\left(I_{1}\right)-2 \xi J^{-3}\left(I_{2}\right)^{3 / 2}+\kappa(J-1),
\end{aligned}
$$

where, following References [4,37], it is then possible to express the first Piola-Kirchhoff stress tensor $\boldsymbol{P}$ in terms of the geometric strains $\{\boldsymbol{F}, \boldsymbol{H}, J\}$ and conjugate stresses $\left\{\boldsymbol{\Sigma}_{\boldsymbol{F}}, \boldsymbol{\Sigma}_{\boldsymbol{H}}, \Sigma_{J}\right\}$ in $(8)$ as

$$
\boldsymbol{P}=\boldsymbol{\Sigma}_{\boldsymbol{F}}+\boldsymbol{\Sigma}_{\boldsymbol{H}} \times \boldsymbol{F}+\Sigma_{J} \boldsymbol{H} .
$$

Finally, the symmetric positive semidefinite Hessian operator $\left[\mathbb{H}_{W}\right]$ of the strain energy density $W(7)$ with respect to the strain measures $\{\boldsymbol{F}, \boldsymbol{H}, J\}$ is computed as

$$
\left[\mathbb{H}_{W}\right]:=\left[\begin{array}{ccc}
\frac{\partial^{2} W}{\partial \boldsymbol{F} \partial \boldsymbol{F}} & \frac{\partial^{2} W}{\partial \boldsymbol{F} \partial \boldsymbol{H}} & \frac{\partial^{2} W}{\partial \boldsymbol{F} \partial J} \\
\frac{\partial^{2} W}{\partial \boldsymbol{H} \partial \boldsymbol{F}} & \frac{\partial^{2} W}{\partial \boldsymbol{H} \partial \boldsymbol{H}} & \frac{\partial^{2} W}{\partial \boldsymbol{H} \partial J} \\
\frac{\partial^{2} W}{\partial J \partial \boldsymbol{F}} & \frac{\partial^{2} W}{\partial J \partial \boldsymbol{H}} & \frac{\partial^{2} W}{\partial J \partial J}
\end{array}\right]=\left[\begin{array}{ccc}
W_{\boldsymbol{F} \boldsymbol{F}} & \mathbf{0} & W_{\boldsymbol{F} J} \\
\mathbf{0} & W_{\boldsymbol{H} \boldsymbol{H}} & W_{\boldsymbol{H} J} \\
W_{J \boldsymbol{F}} & W_{J \boldsymbol{H}} & W_{J J}
\end{array}\right],
$$

with non-zero components

$$
\begin{aligned}
& W_{\boldsymbol{F} \boldsymbol{F}}=2 \varsigma J^{-2 / 3} \mathcal{I} ; \quad W_{\boldsymbol{H} \boldsymbol{H}}=3 \xi J^{-2}\left(I_{2}\right)^{1 / 2}\left[\left(I_{2}\right)^{-1} \boldsymbol{H} \otimes \boldsymbol{H}+\mathcal{I}\right] ; \quad W_{J J}=\gamma+\kappa ; \\
& W_{\boldsymbol{F} J}=-\frac{4 \varsigma}{3} J^{-5 / 3} \boldsymbol{F} ; \quad W_{\boldsymbol{H} J}=-6 \xi J^{-3}\left(I_{2}\right)^{1 / 2} \boldsymbol{H} ; \quad W_{J \boldsymbol{F}}=W_{\boldsymbol{F} J} ; \quad W_{J \boldsymbol{H}}=W_{\boldsymbol{H} J}
\end{aligned}
$$

where $\mathcal{I}$ represents the fourth order identity tensor defined in indicial notation as $[\mathcal{I}]_{i I j J}=$ $\delta_{i j} \delta_{I J}^{4}$ and

$$
\gamma=\frac{10 \varsigma}{9} J^{-8 / 3}\left(I_{1}\right)+6 \xi J^{-4}\left(I_{2}\right)^{3 / 2}
$$

By comparison of the tangent elasticity operator at the initial undeformed configuration with that of the classic linearised elasticity operator [4], appropriate values for the material parameters $\varsigma$ and $\xi$ can be defined in terms of the linearised Lamé coefficient (shear modulus) $\mu$, that is, $2 \varsigma+3 \sqrt{3} \xi=\mu$.

\subsection{Conservation of angular momentum}

The geometry $\boldsymbol{x}$ can be recovered through time integration of the linear momentum $\boldsymbol{p}$ as

$$
\frac{\partial \boldsymbol{x}}{\partial t}=\frac{\boldsymbol{p}}{\rho_{0}} .
$$

\footnotetext{
${ }^{4}$ Capital letters are used to identify Cartesian directions in the initial undeformed configuration and lower case letters are used to identify Cartesian directions in the final configuration [40].
} 
An additional conservation law can be established for the conservation of angular momentum $\boldsymbol{A}=\boldsymbol{x} \times \boldsymbol{p}$, given by

$$
\frac{\partial \boldsymbol{A}}{\partial t}-\mathrm{DIV}(\boldsymbol{x} \times \boldsymbol{P})=\boldsymbol{x} \times \boldsymbol{f}_{0}
$$

In previous publications by the authors $[1-5,32,33,36,41,42]$, a posteriori global projection procedure was used to ensure the satisfaction of (14). In this paper, an alternative local projection is used. Indeed, substitution of equations (1a) and (13) into (14) yields

$$
\mathcal{E}:\left(\boldsymbol{P} \boldsymbol{F}_{\boldsymbol{x}}^{T}\right)=\mathbf{0}
$$

where $\boldsymbol{F}_{\boldsymbol{x}}:=\boldsymbol{\nabla}_{0} \boldsymbol{x}$ and $\mathcal{E}$ represents the third order Levi-Civita (or alternating) tensor. Satisfaction of the constraint (15) will be incorporated into the time integration algorithm (refer to Section 6).

\section{Streamline Upwind Petrov Galerkin (SUPG) algorithm}

The set of local conservation equations described in (1) has been spatially discretised by the authors using a wide variety of stabilised mesh-based techniques (e.g. Finite Element and Finite Volume) $[2-5,32,33,36,41,42]$ and, recently, via a new mesh-free method, namely Jameson-Schmidt-Turkel Smooth Particle Hydrodynamics (JST-SPH) algorithm [1]. In the following section, a novel variationally consistent (residual-based) Streamline Upwind Petrov Galerkin Smooth Particle Hydrodynamics (SUPG-SPH) framework, tailor-made for the mixed-based set $\mathcal{U}=\{\boldsymbol{p}, \boldsymbol{F}, \boldsymbol{H}, J\}$ in (1), is presented.

\subsection{Variationally consistent SUPG}

A SUPG approximation [31] for the above system (1) of conservation variables $\mathcal{U}=$ $\{\boldsymbol{p}, \boldsymbol{F}, \boldsymbol{H}, J\}$ can be established through the definition of appropriate stabilised virtual work conjugates $\delta \mathcal{V}^{s t}:=\delta \mathcal{V}+\delta \mathcal{P}$ (see Eqn. (95) in Reference [5] on pg. 705). These are comprised of the virtual work conjugates of $\mathcal{U}$, namely $\delta \mathcal{V}=\left\{\delta \boldsymbol{v}, \delta \boldsymbol{\Sigma}_{\boldsymbol{F}}, \delta \boldsymbol{\Sigma}_{\boldsymbol{H}}, \delta \Sigma_{J}\right\}$, and the stabilised SUPG contribution $\delta \mathcal{P}$. As already presented in [4] (see Eqn. (48) on pg. 156), the stabilisation contribution $\delta \mathcal{P}$ can be defined as

$$
\delta \mathcal{P}:=\boldsymbol{\tau}^{T} \mathcal{A}_{I}^{T} \frac{\partial \delta \mathcal{V}}{\partial X_{I}}=\left[\begin{array}{c}
-\frac{\tau_{p}}{\rho_{0}}\left(\mathrm{DIV} \delta \boldsymbol{\Sigma}_{\boldsymbol{F}}-\boldsymbol{F} \times \operatorname{CURL} \delta \boldsymbol{\Sigma}_{\boldsymbol{H}}+\boldsymbol{H} \boldsymbol{\nabla}_{0} \delta \Sigma_{J}\right) \\
-\tau_{\boldsymbol{F}}\left(W_{\boldsymbol{F} \boldsymbol{F}}+W_{\boldsymbol{F} J} \otimes \boldsymbol{H}\right): \boldsymbol{\nabla}_{0} \delta \boldsymbol{v} \\
-\tau_{\boldsymbol{H}}\left(W_{\boldsymbol{H} \boldsymbol{H}} \times \boldsymbol{F}+W_{\boldsymbol{H} J} \otimes \boldsymbol{H}\right): \boldsymbol{\nabla}_{0} \delta \boldsymbol{v} \\
-\tau_{J}\left(W_{J \boldsymbol{F}}+W_{J \boldsymbol{H}} \times \boldsymbol{F}+W_{J J} \boldsymbol{H}\right): \boldsymbol{\nabla}_{0} \delta \boldsymbol{v}
\end{array}\right]
$$

where $\mathcal{A}_{I}:=\frac{\partial \mathcal{F}_{I}}{\partial \mathcal{U}}$ represents the flux Jacobian matrix and $W_{A B}$ are Hessian terms defined in (10). Notice that the SUPG contribution (16) includes the so-called intrinsic time-scale ma$\operatorname{trix} \boldsymbol{\tau}$, which is key for the success of a stabilised formulation [43]. Specifically, a diagonal matrix $\boldsymbol{\tau}$ already presented in [2-5] and comprised in general of the coefficients $\left\{\tau_{\boldsymbol{p}}, \tau_{\boldsymbol{F}}, \tau_{\boldsymbol{H}}, \tau_{J}\right\}$, is used in this paper. Finally, the use of $\mathcal{U}$ and $\delta \mathcal{V}^{s t}$ yields the following generic (weak) variational statement $[2-4,31]$

$$
\mathcal{A}_{\mathrm{Gal}}(\mathcal{U}, \delta \mathcal{V})+\mathcal{A}_{\mathrm{SUPG}}(\mathcal{U}, \delta \mathcal{V})=0
$$


where

$$
\begin{aligned}
\mathcal{A}_{\mathrm{Gal}}(\mathcal{U}, \delta \mathcal{V}) & :=\int_{V} \delta \mathcal{V} \bullet \frac{\partial \mathcal{U}}{\partial t} d V-\int_{V} \mathcal{F}_{I} \bullet \frac{\partial \delta \mathcal{V}}{\partial X_{I}} d V \\
& +\int_{\partial V} \delta \mathcal{V} \bullet \mathcal{F}_{I} N_{I} d A-\int_{V} \delta \mathcal{V} \bullet \mathcal{S} d V \\
\mathcal{A}_{\mathrm{SUPG}}(\mathcal{U}, \delta \mathcal{V}) & :=-\int_{V} \delta \mathcal{P} \bullet \mathcal{R} d V
\end{aligned}
$$

with the symbol $\bullet$ used to denote the inner (dual) product of work conjugate pairs and $N_{I}$ the $I$-th component of the outward unit normal vector $\boldsymbol{N}$ on the boundary $\partial V$. In addition, the so-called residual term $\mathcal{R}$ of each conservation law in (1), featuring in (18b), can be expanded as

$$
\mathcal{R}=\left[\begin{array}{c}
\mathcal{R}_{p} \\
\mathcal{R}_{\boldsymbol{F}} \\
\mathcal{R}_{\boldsymbol{H}} \\
\mathcal{R}_{J}
\end{array}\right]=\left[\begin{array}{c}
\operatorname{DIVP}+\boldsymbol{f}_{0}-\frac{\partial \boldsymbol{p}}{\partial t} \\
\boldsymbol{\nabla}_{0}\left(\frac{\boldsymbol{p}}{\rho_{0}}\right)-\frac{\partial \boldsymbol{F}}{\partial t} \\
\boldsymbol{F} \times \boldsymbol{\nabla}_{0}\left(\frac{\boldsymbol{p}}{\rho_{0}}\right)-\frac{\partial \boldsymbol{H}}{\partial t} \\
\boldsymbol{H}: \boldsymbol{\nabla}_{0}\left(\frac{\boldsymbol{p}}{\rho_{0}}\right)-\frac{\partial J}{\partial t}
\end{array}\right] .
$$

Notice that in above variational statement (17), the term $\mathcal{A}_{\text {Gal }}$ corresponds to the standard Bubnov-Galerkin contribution and $\mathcal{A}_{\mathrm{SUPG}}$ is the SUPG stabilisation contribution, the latter needed to counterbalance the negative diffusion introduced by the standard Bubnov-Galerkin approximation [44].

Substitution of expression (16) into (17) enables the (stabilised) weak variational statement for the conservation of linear momentum (1a) to be obtained as

$$
\begin{aligned}
0 & =\underbrace{\int_{V} \delta \boldsymbol{v} \cdot \frac{\partial \boldsymbol{p}}{\partial t} d V+\int_{V} \boldsymbol{P}: \boldsymbol{\nabla}_{0} \delta \boldsymbol{v} d V-\int_{V} \delta \boldsymbol{v} \cdot \boldsymbol{f}_{0} d V-\int_{\partial V} \delta \boldsymbol{v} \cdot \boldsymbol{t}_{B} d A}_{\mathcal{A}_{\mathrm{Gal}}^{p}} \\
& +\underbrace{\int_{V} \boldsymbol{P}_{\mathrm{fine}}: \boldsymbol{\nabla}_{0} \delta \boldsymbol{v} d V}_{\mathcal{A}_{\mathrm{SUPG}}^{\boldsymbol{p}}},
\end{aligned}
$$

where $\boldsymbol{t}_{B}$ represents the (applied) boundary traction vector and $\boldsymbol{P}_{\text {fine }}$ is the so-called fine-scale first Piola-Kirchhoff stress defined as

$$
\begin{aligned}
\boldsymbol{P}_{\text {fine }} & =\tau_{\boldsymbol{F}}\left(W_{\boldsymbol{F} \boldsymbol{F}}+W_{\boldsymbol{F} J} \otimes \boldsymbol{H}\right): \boldsymbol{\mathcal { R }}_{\boldsymbol{F}}+\tau_{\boldsymbol{H}}\left(W_{\boldsymbol{H} \boldsymbol{H}} \times \boldsymbol{F}+W_{\boldsymbol{H} J} \otimes \boldsymbol{H}\right): \boldsymbol{\mathcal { R }}_{\boldsymbol{H}} \\
& +\tau_{J}\left(W_{J \boldsymbol{F}}+W_{J \boldsymbol{H}} \times \boldsymbol{F}+W_{J J} \boldsymbol{H}\right) \mathcal{R}_{J}
\end{aligned}
$$

given in terms of the three residual components $\left\{\boldsymbol{\mathcal { R }}_{\boldsymbol{F}}, \boldsymbol{\mathcal { R }}_{\boldsymbol{H}}, \mathcal{R}_{J}\right\}$, the three stabilisation coefficients $\left\{\tau_{\boldsymbol{F}}, \tau_{\boldsymbol{H}}, \tau_{J}\right\}$ and some of the Hessian components $W_{A B}$. It is instructive to re-write above expression (21) (refer to (9)) as

$$
\boldsymbol{P}_{\text {fine }}=\Sigma_{\boldsymbol{F}}^{\text {fine }}+\boldsymbol{\Sigma}_{\boldsymbol{H}}^{\text {fine }} \times \boldsymbol{F}+\Sigma_{J}^{\text {fine }} \boldsymbol{H}
$$


where the fine-scale conjugate stresses $\left\{\Sigma_{\boldsymbol{F}}^{\mathrm{fine}}, \boldsymbol{\Sigma}_{\boldsymbol{H}}^{\mathrm{fine}}, \Sigma_{J}^{\mathrm{fine}}\right\}$ are given by

$$
\begin{aligned}
& \Sigma_{\boldsymbol{F}}^{\text {fine }}=\tau_{\boldsymbol{F}} W_{\boldsymbol{F} \boldsymbol{F}}: \mathcal{R}_{\boldsymbol{F}}+\tau_{J} W_{J \boldsymbol{F}} \mathcal{R}_{J} \\
& \Sigma_{\boldsymbol{H}}^{\text {fine }}=\tau_{\boldsymbol{H}} W_{\boldsymbol{H} \boldsymbol{H}}: \boldsymbol{\mathcal { R }}_{\boldsymbol{H}}+\tau_{J} W_{J \boldsymbol{H}} \mathcal{R}_{J} ; \\
& \Sigma_{J}^{\text {fine }}=\tau_{\boldsymbol{F}} W_{\boldsymbol{F} J}: \boldsymbol{\mathcal { R }}_{\boldsymbol{F}}+\tau_{\boldsymbol{H}} W_{\boldsymbol{H} J}: \mathcal{R}_{\boldsymbol{H}}+\tau_{J} W_{J J} \mathcal{R}_{J},
\end{aligned}
$$

with : denoting the double contraction tensor operation [40]. Choosing $\tau_{\boldsymbol{H}}=0$ and $\tau_{J}=0$, the fine-scale stress $\boldsymbol{P}_{\text {fine }}(21)$ results in

$$
\boldsymbol{P}_{\text {fine }}=\tau_{\boldsymbol{F}}\left(W_{\boldsymbol{F F}}+W_{\boldsymbol{F} J} \otimes \boldsymbol{H}\right): \boldsymbol{\mathcal { R }}_{\boldsymbol{F}},
$$

defined in terms of a single stabilisation coefficient $\tau_{\boldsymbol{F}}$ and the residual component $\boldsymbol{\mathcal { R }}_{\boldsymbol{F}}$ expressing the difference between the time rate of the fibre map and its evaluation in terms of the material gradient of the velocity (19). An even simpler approach can be postulated where the Hessian components $W_{A B}$ in (23) are replaced by those of a simplified hyperelastic model defined by

$$
\widetilde{W}(\boldsymbol{F})=\frac{\gamma}{2} I_{1}
$$

where $\gamma$ is a user-defined material constant, usually taken in the neighbourhood of the bulk modulus $\kappa$ of the material. In this case, all Hessian components vanish but $\widetilde{W}_{\boldsymbol{F} F}$, which remains constant throughout the deformation process, namely $\widetilde{W}_{\boldsymbol{F} \boldsymbol{F}}=\gamma \mathcal{I}$, which upon substitution into (24) yields

$$
\boldsymbol{P}_{\text {fine }}=\tau_{\boldsymbol{F}} \gamma \boldsymbol{\mathcal { R }}_{\boldsymbol{F}} .
$$

For the examples presented in this paper, this alternative stabilisation (26) has been seen to be as robust (yet computationally more efficient) as (24). Following [2-5] and in order to reduce the level of implicitness of the formulation, the above fine-scale stress $\boldsymbol{P}_{\text {fine }}$ can be further enhanced by introducing the time integrated residual $\mathcal{R}_{\boldsymbol{F}}^{\boldsymbol{x}}:=\boldsymbol{F}_{\boldsymbol{x}}-\boldsymbol{F}$ to result in

$$
\boldsymbol{P}_{\text {fine }}=\tau_{\boldsymbol{F}} \gamma \boldsymbol{\mathcal { R }}_{\boldsymbol{F}}+\zeta_{\boldsymbol{F}} \alpha \boldsymbol{\mathcal { R }}_{\boldsymbol{F}}^{\boldsymbol{x}},
$$

where $\alpha$ is another user-defined material constant (typically in the range of the shear modulus $\mu)$ and $\zeta_{\boldsymbol{F}}$ is a dimensionless stabilisation parameter in the range of $[0,0.5]$ [2].

\section{Remark 1:}

Notice that combination of the second and fifth terms on the right hand side of equation (20) enables the definition of the stabilised first Piola-Kirchhoff stress tensor $\boldsymbol{P}_{\text {st }}$, additively decomposed into a Bubnov-Galerkin (or coarse-scale) contribution $\boldsymbol{P}$ and the SUPG (or fine-scale) contribution $\boldsymbol{P}_{\text {fine }}(27)$, namely $\boldsymbol{P}_{\text {st }}:=\boldsymbol{P}+\boldsymbol{P}_{\text {fine }}$.

Alternatively, if a Variational Multi-Scale (VMS) approach [2-5, 45-48] is followed, the stabilised first Piola-Kirchhoff stress tensor $\boldsymbol{P}_{\text {st }}$ is obtained as a function of the extended set of stabilised strains, namely $\boldsymbol{P}_{\text {st }}:=\boldsymbol{P}\left(\boldsymbol{F}_{\text {st }}, \boldsymbol{H}_{\text {st }}, J_{\text {st }}\right)[2-5]$, with $\left\{\boldsymbol{F}_{\text {st }}, \boldsymbol{H}_{\text {st }}, J_{\text {st }}\right\}$ defined as

$$
\begin{aligned}
\boldsymbol{F}_{\mathrm{st}} & :=\boldsymbol{F}+\tau_{\boldsymbol{F}} \boldsymbol{\mathcal { R }}_{\boldsymbol{F}}+\xi_{\boldsymbol{F}} \boldsymbol{\mathcal { R }}_{\boldsymbol{F}}^{\boldsymbol{x}} ; \\
\boldsymbol{H}_{\mathrm{st}} & :=\boldsymbol{H}+\tau_{\boldsymbol{H}} \boldsymbol{\mathcal { R }}_{\boldsymbol{H}}+\xi_{\boldsymbol{H}} \boldsymbol{\mathcal { R }}_{\boldsymbol{H}}^{\boldsymbol{x}} \\
J_{\mathrm{st}} & :=J+\tau_{J} \boldsymbol{\mathcal { R }}_{J}+\xi_{J} \boldsymbol{\mathcal { R }}_{J}^{\boldsymbol{x}},
\end{aligned}
$$

with the new time integrated residuals $\boldsymbol{\mathcal { R }}_{\boldsymbol{H}}^{\boldsymbol{x}}$ and $\mathcal{R}_{J}^{\boldsymbol{x}}$ defined as $\boldsymbol{\mathcal { R }}_{\boldsymbol{H}}^{\boldsymbol{x}}:=\frac{1}{2}\left(\boldsymbol{F}_{\boldsymbol{x}} \times \boldsymbol{F}_{\boldsymbol{x}}\right)-\boldsymbol{H}$ and $\mathcal{R}_{J}^{\boldsymbol{x}}:=\operatorname{det}\left(\boldsymbol{F}_{\boldsymbol{x}}\right)-J$, respectively. This alternative VMS approach $(28 \mathrm{a}, \mathrm{b}, \mathrm{c})$ has been 
thoroughly explored by the authors in recent publications $[2-5,33]$ in the context of Finite Element formulations.

Substitution of (16) into (17) enables the (stabilised) weak statement for the conservation of the strain measures $\{\boldsymbol{F}, \boldsymbol{H}, J\}$ to be obtained as follows

$$
\begin{aligned}
& \underbrace{\int_{V} \delta \boldsymbol{\Sigma}_{\boldsymbol{F}}:\left[\frac{\partial \boldsymbol{F}}{\partial t}-\boldsymbol{\nabla}_{0}\left(\frac{\boldsymbol{p}}{\rho_{0}}\right)\right] d V}_{\mathcal{A}_{\mathrm{Gal}}^{F}} \underbrace{+\int_{V} \operatorname{DIV} \delta \boldsymbol{\Sigma}_{\boldsymbol{F}} \cdot\left(\frac{\tau_{\boldsymbol{p}}}{\rho_{0}} \boldsymbol{\mathcal { R }}_{\boldsymbol{p}}\right) d V}_{\mathcal{A}_{\mathrm{Gal}}^{H}}=0 ; \\
& \underbrace{\int_{V} \delta \boldsymbol{\Sigma}_{\boldsymbol{H}}:\left[\frac{\partial \boldsymbol{H}}{\partial t}-\boldsymbol{F} \times \boldsymbol{\nabla}_{0}\left(\frac{\boldsymbol{p}}{\rho_{0}}\right)\right] d V}_{\mathcal{A}_{\mathrm{Gal}}^{J}}-\underbrace{-\int_{V} \operatorname{CURL} \delta \boldsymbol{\Sigma}_{\boldsymbol{H}}:\left(\frac{\tau_{\boldsymbol{p}}}{\rho_{0}} \boldsymbol{\mathcal { R }}_{\boldsymbol{p}} \times \boldsymbol{F}\right) d V}_{\mathcal{A}_{\mathrm{SUPG}}^{H}}=0 ; \\
& \underbrace{\int_{V} \delta \Sigma_{J}\left[\frac{\partial J}{\partial t}-\boldsymbol{H}: \boldsymbol{\nabla}_{0}\left(\frac{\boldsymbol{p}}{\rho_{0}}\right)\right] d V}_{\mathcal{A}_{\mathrm{SUPG}}^{J}}+\underbrace{\int_{V}^{\boldsymbol{\nabla}_{0} \delta \Sigma_{J} \cdot\left(\frac{\tau_{\boldsymbol{p}}}{\rho_{0}} \boldsymbol{H}^{T} \boldsymbol{\mathcal { R }}_{\boldsymbol{p}}\right) d V}}_{V}=0 .
\end{aligned}
$$

Finally, discrete satisfaction of the involutions (2) can be achieved by neglecting the terms $\mathcal{A}_{\mathrm{SUPG}}^{\boldsymbol{F}}$ in (29a) and $\mathcal{A}_{\mathrm{SUPG}}^{\boldsymbol{H}}$ in $(29 \mathrm{~b})$ (see $[4,5]$ for further details). Thus, only the SUPG term $\mathcal{A}_{\mathrm{SUPG}}^{J}$ (and $\mathcal{A}_{\mathrm{SUPG}}^{p}$ in (20)) will be considered henceforth.

\section{Smooth Particle Hydrodynamics (SPH) approximation}

Before presenting the spatial discretisation of the mixed-based system $\{\boldsymbol{p}, \boldsymbol{F}, \boldsymbol{H}, J\}$, we very briefly summarise some important concepts of SPH kernel interpolation and derivatives adopted in this work. Given a set of particles $a(b)$ defined by its material position $\boldsymbol{X}_{a}\left(\boldsymbol{X}_{b}\right)$, the same corrected kernel (or smoothing function) $\tilde{W}$ as that reported in [49] (see Section 4.2 on pg. 105-106) is followed in the present manuscript. This ensures zero-th and first order consistency. Additionally, and contrary to the JST-SPH framework in [1] (see Eqn. (21) on pg. 77 in Reference [1]), the discrete approximation of the material gradient of any arbitrary vector function $\boldsymbol{f}$ at position $\boldsymbol{X}_{a}$ is carried out via the use of the "Corrected Gradient of a Corrected Kernel $\tilde{\boldsymbol{\nabla}}_{0} \tilde{\mathrm{W}}^{\prime \prime}[49]$ as

$$
\nabla_{0} \boldsymbol{f}\left(\boldsymbol{X}_{a}\right) \approx \sum_{b \in \Lambda_{a}^{b}} \boldsymbol{f}_{b} \otimes \boldsymbol{G}_{b}\left(\boldsymbol{X}_{a}\right)
$$

where $\boldsymbol{G}_{b}\left(\boldsymbol{X}_{a}\right):=V_{b} \tilde{\boldsymbol{\nabla}}_{0} \tilde{\mathrm{W}}_{b}\left(\boldsymbol{X}_{a}\right)$ and $V_{b}$ represents the volume associated with particle $b$ belonging to the support $\Lambda_{a}^{b}$ of particle $a$.

\subsection{Bubnov-Galerkin contribution}

Following the same procedure employed in any standard SPH spatial approximation [1,

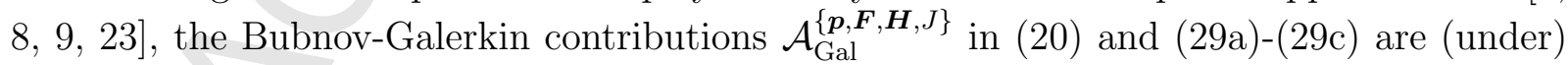
integrated at a primary set of (Galerkin) particles $[1,6,8,10,50,51]$. Using a kernel interpolation $\tilde{W}$ (see Section 3.2 on pg. 77 in Reference [1]) and its gradient evaluation as in 
(30), terms $\mathcal{A}_{\mathrm{Gal}}^{\{\boldsymbol{p}, \boldsymbol{F}, \boldsymbol{H}, J\}}$ result in the following (particle) discrete system of equations

$$
\begin{aligned}
\frac{d \boldsymbol{p}_{a}}{d t} & =\boldsymbol{E}_{a}-\boldsymbol{T}_{a} ; \\
\frac{d \boldsymbol{F}_{a}}{d t} & =\sum_{b \in \Lambda_{a}^{b}} \frac{\boldsymbol{p}_{b}}{\rho_{0}} \otimes \boldsymbol{G}_{b}\left(\boldsymbol{X}_{a}\right) ; \\
\frac{d \boldsymbol{H}_{a}}{d t} & =\boldsymbol{F}_{a} \times \sum_{b \in \Lambda_{a}^{b}} \frac{\boldsymbol{p}_{b}}{\rho_{0}} \otimes \boldsymbol{G}_{b}\left(\boldsymbol{X}_{a}\right) ; \\
\frac{d J_{a}}{d t} & =\boldsymbol{H}_{a}: \sum_{b \in \Lambda_{a}^{b}} \frac{\boldsymbol{p}_{b}}{\rho_{0}} \otimes \boldsymbol{G}_{b}\left(\boldsymbol{X}_{a}\right) .
\end{aligned}
$$

Here, the nodal external $\boldsymbol{E}_{a}$ and internal $\boldsymbol{T}_{a}$ force vectors are defined as

$$
\boldsymbol{E}_{a}=\frac{A_{a}}{V_{a}} \boldsymbol{t}_{a}+\boldsymbol{f}_{0}^{a} ; \quad \boldsymbol{T}_{a}=\sum_{b \in \Lambda_{a}^{b}} \frac{V_{b}}{V_{a}} \boldsymbol{P}_{b} \boldsymbol{G}_{a}\left(\boldsymbol{X}_{b}\right),
$$

with $\boldsymbol{P}_{b}:=\boldsymbol{P}\left(\boldsymbol{F}_{b}, \boldsymbol{H}_{b}, J_{b}\right)$ and $A_{a}$ and $\boldsymbol{t}_{a}$ the tributary area and traction vector of those particles placed at the boundary, the latter being computed directly from the given traction boundary conditions $[32,41,42]$. It is worth mentioning that, the above internal force representation $\boldsymbol{T}_{a}(32 \mathrm{~b})$ satisfies the global conservation of linear momentum, that is $\sum_{a} V_{a} \boldsymbol{T}_{a}=\mathbf{0}$ (see Remark 4 on pg. 79 in Reference [1]).

As it is well known [1-5], the above discrete mixed-based system (31) suffers from severe numerical instabilities $[16,20,23,52]$, especially in nearly incompressible scenarios. The additional SUPG contributions $\mathcal{A}_{\mathrm{SUPG}}^{\{\boldsymbol{p}, J\}}$ in equations (20) and (29c), crucial to circumvent this shortcoming, are presented in the following section.

\subsection{SUPG contribution}

Evaluation of the terms $\mathcal{A}_{\text {SUPG }}^{\{\boldsymbol{p}, J\}}$ in (20) and (29c) at the primary set of particles does not introduce any effect, given the residual-based nature of the SUPG stabilisation (e.g. the discrete residuals are strictly zero when evaluated at the primary set of particles). Hence, and taking inspiration from the Dual Particle Method, [25, 53-55], a secondary set of particles is employed in order to account for the effect of these SUPG terms.

In general, there are several options to define the positions of this secondary set of particles. One of the most commonly used approaches in mesh-free methods (especially in the context of Element Free Galerkin method $[56,57]$ ) is to allocate these particles coinciding with the Gauss quadrature points of an underlying fictitious finite element mesh. Following this approach, two simple alternative particle arrangements are used in this paper, namely: SUPG-SPH-H1, where the secondary particles are placed at the centroids of the hexahedral elements of an underlying hexahedral mesh, and SUPG-SPH-H6, where the secondary particles are placed at the centroids of tetrahedral elements generated after splitting of an initial underlying hexahedral mesh (i.e. one hexahedral element leads to six tetrahedral elements) (refer to Figure 2). The secondary set of particles are only used for the evaluation of the SUPG terms. It is worth emphasising that, as opposed to Reference [58], stability of the algorithm (Ladyzhenskaya-Babuska-Brezzi (LBB) condition) does not rely upon a specifically designed particle arrangement. The automated generation of the secondary set of particles, without resorting to an underlying (fictitious) mesh, is not investigated as part of this paper. 


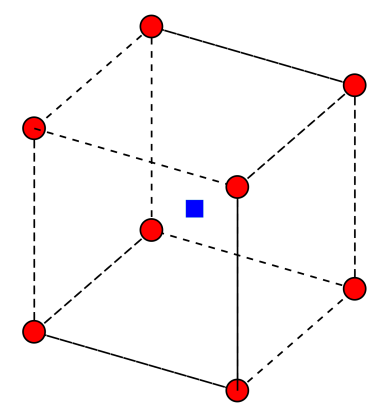

(a)

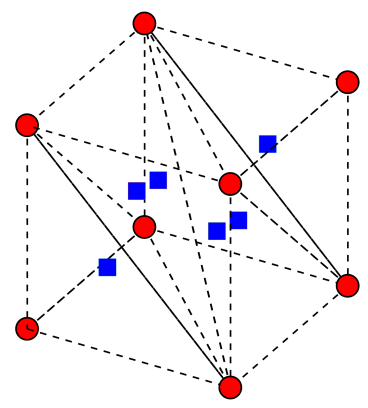

(b)

Figure 2: The positions of the primary and secondary set of particles in three dimensional hexahedral fictitious mesh with: (a) 1 Gauss quadrature point; and (b) 6 Gauss quadrature points. The primary set of particles is placed at the vertices (nodes), whereas the secondary set of particles coincides with the Gauss quadrature points of the underlying mesh.

Nodal integration of the SUPG term $\mathcal{A}_{\mathrm{SUPG}}^{p}(20)$ at a secondary set of particles $q$ gives

$$
\begin{aligned}
\mathcal{A}_{\mathrm{SUPG}}^{p} & :=-\int_{V} \boldsymbol{P}_{\text {fine }}: \nabla_{0} \delta \boldsymbol{v} d V \\
& \approx-\sum_{q} V_{q} \boldsymbol{P}_{\text {fine }}\left(\boldsymbol{X}_{q}\right): \nabla_{0} \delta \boldsymbol{v}\left(\boldsymbol{X}_{q}\right),
\end{aligned}
$$

where $\boldsymbol{P}_{\text {fine }}\left(\boldsymbol{X}_{q}\right):=\tau_{\boldsymbol{F}} \gamma \boldsymbol{\mathcal { R }}_{\boldsymbol{F}}\left(\boldsymbol{X}_{q}\right)+\zeta_{\boldsymbol{F}} \alpha \boldsymbol{\mathcal { R }}_{\boldsymbol{F}}^{\boldsymbol{x}}\left(\boldsymbol{X}_{q}\right)$ (refer to (27)). With the aid of the material gradient evaluation described in (30), equation (33) can equivalently be written in terms of a dissipative force vector $\mathcal{D}\left(\boldsymbol{p}_{a}\right)$ as

$$
\begin{aligned}
\mathcal{A}_{\mathrm{SUPG}}^{p} & \approx-\sum_{q} V_{q} \boldsymbol{P}_{\text {fine }}\left(\boldsymbol{X}_{q}\right):\left(\sum_{a \in \Lambda_{q}^{a}} \delta \boldsymbol{v}_{a} \otimes \boldsymbol{G}_{a}\left(\boldsymbol{X}_{q}\right)\right) \\
& =\sum_{a} V_{a} \delta \boldsymbol{v}_{a} \cdot(\underbrace{-\sum_{q \in \Lambda_{a}^{q}} \frac{V_{q}}{V_{a}} \boldsymbol{P}_{\text {fine }}\left(\boldsymbol{X}_{q}\right) \boldsymbol{G}_{a}\left(\boldsymbol{X}_{q}\right)}_{\mathcal{D}\left(\boldsymbol{p}_{a}\right)}) .
\end{aligned}
$$

Finally, the SUPG contribution $\mathcal{A}_{\text {SUPG }}^{J}$ (associated with spurious pressure mechanisms) 
in $(29 \mathrm{c})$ yields

$$
\begin{aligned}
\mathcal{A}_{\mathrm{SUPG}}^{J} & :=-\int_{V} \boldsymbol{\nabla}_{0} \delta \Sigma_{J} \cdot\left(\frac{\tau_{\boldsymbol{p}}}{\rho_{0}} \boldsymbol{H}^{T} \boldsymbol{\mathcal { R }}_{\boldsymbol{p}}\right) d V \\
& \approx-\frac{\tau_{\boldsymbol{p}}}{\rho_{0}} \sum_{q} V_{q} \boldsymbol{\nabla}_{0} \delta \Sigma_{J}\left(\boldsymbol{X}_{q}\right) \cdot\left(\boldsymbol{H}^{T}\left(\boldsymbol{X}_{q}\right) \boldsymbol{\mathcal { R }}_{\boldsymbol{p}}\left(\boldsymbol{X}_{q}\right)\right) \\
& \approx-\frac{\tau_{\boldsymbol{p}}}{\rho_{0}} \sum_{q} V_{q}\left(\sum_{a \in \Lambda_{q}^{a}} \delta \Sigma_{J}^{a} \boldsymbol{G}_{a}\left(\boldsymbol{X}_{q}\right)\right) \cdot\left(\boldsymbol{H}^{T}\left(\boldsymbol{X}_{q}\right) \boldsymbol{\mathcal { R }}_{\boldsymbol{p}}\left(\boldsymbol{X}_{q}\right)\right) \\
& =\sum_{a} V_{a} \delta \Sigma_{J}^{a}[\underbrace{-\frac{\tau_{\boldsymbol{p}}}{\rho_{0}}\left(\sum_{q \in \Lambda_{a}^{q}} \frac{V_{q}}{V_{a}}\left(\boldsymbol{H}^{T}\left(\boldsymbol{X}_{q}\right) \boldsymbol{\mathcal { R }}_{\boldsymbol{p}}\left(\boldsymbol{X}_{q}\right)\right) \cdot \boldsymbol{G}_{a}\left(\boldsymbol{X}_{q}\right)\right)}_{\mathcal{D}\left(J_{a}\right)}] .
\end{aligned}
$$

Notice that both SUPG stabilising terms, namely $\mathcal{D}\left(\boldsymbol{p}_{a}\right)$ and $\mathcal{D}\left(J_{a}\right)$, are introduced through a variationally consistent framework. Hence, both naturally satisfy the global conservation requirement, that is $\sum_{a} V_{a} \mathcal{D}\left(\boldsymbol{p}_{a}\right)=\mathbf{0}$ and $\sum_{a} V_{a} \mathcal{D}\left(J_{a}\right)=0$. This is a crucial advantage with respect to the JST stabilisation algorithm in Reference [1] (see Eqns. (49a) and (51) on pg. 83 in Reference [1]), where a posteriori (least-square) projection procedure was needed for global conservation.

\section{Complete SUPG-SPH algorithm}

Addition of the discrete variationally consistent SUPG contributions (see (34) and (35)) to the Bubnov-Galerkin discrete expressions (31a-31d), finally yields the complete semi-discrete set of governing equations for $\{\boldsymbol{p}, \boldsymbol{F}, \boldsymbol{H}, J\}$ as

$$
\begin{aligned}
\frac{d \boldsymbol{p}_{a}}{d t} & =\boldsymbol{E}_{a}-\boldsymbol{T}_{a}+\mathcal{D}\left(\boldsymbol{p}_{a}\right) ; \\
\frac{d \boldsymbol{F}_{a}}{d t} & =\sum_{b \in \Lambda_{a}^{b}} \frac{\boldsymbol{p}_{b}}{\rho_{0}} \otimes \boldsymbol{G}_{b}\left(\boldsymbol{X}_{a}\right) ; \\
\frac{d \boldsymbol{H}_{a}}{d t} & =\boldsymbol{F}_{a} \times \sum_{b \in \Lambda_{a}^{b}} \frac{\boldsymbol{p}_{b}}{\rho_{0}} \otimes \boldsymbol{G}_{b}\left(\boldsymbol{X}_{a}\right) ; \\
\frac{d J_{a}}{d t} & =\boldsymbol{H}_{a}: \sum_{b \in \Lambda_{a}^{b}} \frac{\boldsymbol{p}_{b}}{\rho_{0}} \otimes \boldsymbol{G}_{b}\left(\boldsymbol{X}_{a}\right)+\mathcal{D}\left(J_{a}\right),
\end{aligned}
$$

with the stabilising terms $\left\{\boldsymbol{D}\left(\boldsymbol{p}_{a}\right), \mathcal{D}\left(J_{a}\right)\right\}$ and the internal and external force vectors $\left\{\boldsymbol{T}_{a}, \boldsymbol{E}_{a}\right\}$ defined in Section 4. Notice that the SUPG stabilisation is only applied to the linear momentum evolution $\mathcal{D}\left(\boldsymbol{p}_{a}\right)$ (36a) and the volume map evolution $\mathcal{D}\left(J_{a}\right)(36 \mathrm{~d})$. The former alleviates the appearance of spurious zero-energy (hourglass-like [10]) modes whereas the latter removes pressure instabilities in nearly incompressible scenarios [3].

Time integration of the geometry $\boldsymbol{x}$ and the above semi-discrete system (36) is carried out monolithically via a one-step two-stage Total Variation Diminishing Runge-Kutta (TVD-RK) method, thoroughly reported by the authors in [1] and references therein. 
Remark 2:

It is interesting to emphasise two appealing features of the proposed SUPG algorithm with respect to the JST algorithm developed by the authors in [1]. First, the variationally consistent nature of the SUPG stabilisation eliminates the a posteriori projection procedure (see Eqns. (49a) and (51) on pg. 83 in Reference [1]) used for global conservation in the JST algorithm. Second, the SUPG algorithm does not require the computationally intensive evaluation and the a priori corrections (for consistency purposes) of the harmonic and biharmonic operators, needed in the case of the JST algorithm.

\section{Local angular momentum preserving algorithm}

As described in Section 2 (refer to (15)), the resulting SUPG-SPH algorithm intrinsically fulfils conservation of angular momentum provided that $\boldsymbol{P} \boldsymbol{F}_{\boldsymbol{x}}{ }^{T}$ is a symmetric tensor. This is automatically satisfied in displacement-based formulations [40]. However, this is generally not the case in mixed formulations, as that presented in this paper, where the first PiolaKirchhoff stress tensor is a function of a set of (weakly related) geometric strains variables, that is $\boldsymbol{P}=\boldsymbol{P}(\boldsymbol{F}, \boldsymbol{H}, J)$ (see Section 2.1 on pg. 75 in Reference [1]).

In contrast to the global projection proposed by the authors in previous publications $[1,2,32,36,41,42]$, a locally preserving angular momentum algorithm is adopted in this paper. For this, constraint (15) is enforced at every particle $a$ at each stage of the one-step two-stage Runge Kutta time integrator (see Eqn. (43) on pg. 82 in Reference [1]) [42]

$$
\mathcal{E}:\left(\boldsymbol{P}_{a}^{\chi} \boldsymbol{F}_{\mathscr{X}_{a}}^{T}\right)=\mathbf{0}, \quad \forall \chi=\{n, \star\} ;
$$

where

$$
\mathscr{X}_{a}=\left\{\begin{array}{cl}
\boldsymbol{x}_{a}^{n}, & \text { if } \chi=n \\
\boldsymbol{x}_{a}^{n+1}:=\boldsymbol{x}_{a}^{n}+\frac{\Delta t}{2 \rho_{0}}\left(\boldsymbol{p}_{a}^{n}+\boldsymbol{p}_{a}^{\star}\right), & \text { if } \chi=\star
\end{array} .\right.
$$

A least-square minimisation procedure is used to obtain a modified set of particle stresses $\boldsymbol{P}_{a}^{C}$ that satisfy above condition (37). This can be achieved by computing the minimum of the following functional $[36,42]$ (where time arguments have been ignored for brevity)

$$
\Pi\left(\boldsymbol{P}_{a}^{C}, \boldsymbol{\lambda}_{a}\right)=\frac{1}{2}\left(\boldsymbol{P}_{a}^{C}-\boldsymbol{P}\right):\left(\boldsymbol{P}_{a}^{C}-\boldsymbol{P}_{a}\right)+\boldsymbol{\lambda}_{a} \cdot\left[\mathcal{E}:\left(\boldsymbol{P}_{a}^{C} \boldsymbol{F}_{\mathscr{X}_{a}}^{T}\right)\right],
$$

where $\boldsymbol{P}_{a}^{C}$ represents the (corrected) projected first Piola-Kirchhoff stress tensor and $\boldsymbol{\lambda}_{a}$ a Lagrange multiplier vector. The stationary conditions of the above functional (39) with respect to $\boldsymbol{\lambda}_{a}$ and $\boldsymbol{P}_{a}^{C}$ render

$$
\mathcal{E}:\left(\boldsymbol{P}_{a}^{C} \boldsymbol{F}_{\mathscr{X}_{a}}^{T}\right)=\mathbf{0} ; \quad \boldsymbol{P}_{a}^{C}=\boldsymbol{P}_{a}+\boldsymbol{\lambda}_{a} \times \boldsymbol{F}_{\mathscr{X}_{a}}
$$

Substitution of expression (40b) into (40a) for $\boldsymbol{P}_{a}^{C}$ enables the nodal Lagrange multiplier $\boldsymbol{\lambda}_{a}$ to be evaluated as

$$
\boldsymbol{\lambda}_{a}=\mathcal{L}^{-1}\left[\mathcal{E}:\left(\boldsymbol{P}_{a} \boldsymbol{F}_{\mathscr{X}_{a}}^{T}\right)\right] ; \quad \mathcal{L}=\left(\operatorname{tr} \boldsymbol{b}_{\mathscr{X}_{a}}\right) \boldsymbol{I}-\boldsymbol{b}_{\mathscr{X}_{a}} ;
$$

where $\boldsymbol{b}_{\mathscr{X}_{a}}=\boldsymbol{F}_{\mathscr{X}_{a}} \boldsymbol{F}_{\mathscr{X}_{a}}^{T}$. Analogously, the same projection procedure has to be applied for the evaluation of the projected fine-scale stress tensor $\boldsymbol{P}_{\text {fine }}^{C}(27)$. 


\section{Algorithmic description}

For ease of understanding, Algorithm 1 summarises the complete algorithmic description of the Streamline Upwind Petrov Galerkin Smooth Particle Hydrodynamics (SUPG-SPH) mixed-based $\{\boldsymbol{p}, \boldsymbol{F}, \boldsymbol{H}, J\}$ methodology, with all the necessary numerical ingredients. Notice that simpler $\{\boldsymbol{p}, \boldsymbol{F}\},\{\boldsymbol{p}, \boldsymbol{F}, J\}$ and $\{\boldsymbol{p}, J\}$ versions of the algorithm can be easily obtained by neglecting the relevant geometric conservation laws (i.e. (36c) and/or (36d)).

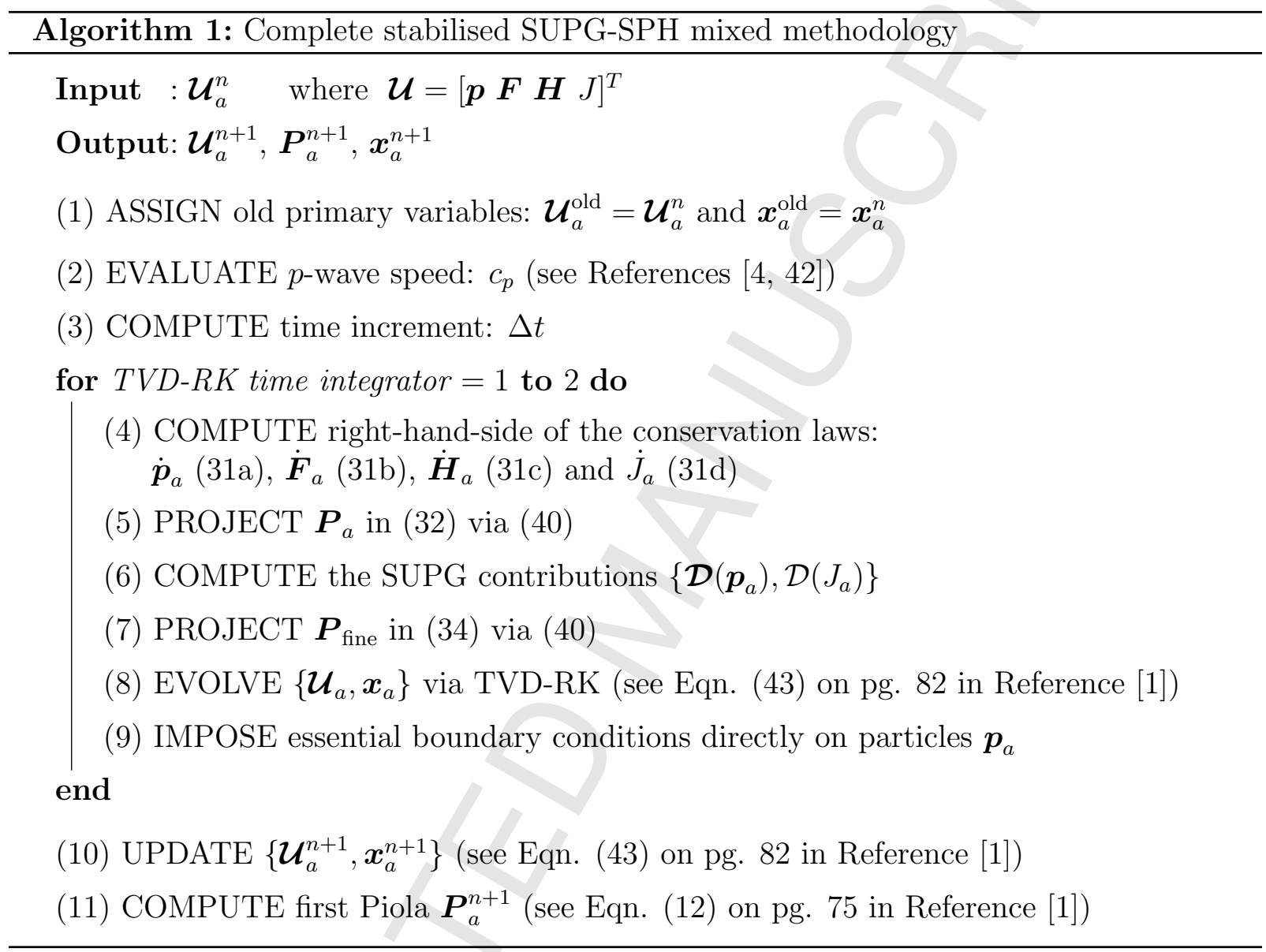

\section{Numerical examples}

In this section, a series of numerical examples are presented in order to assess the robustness, effectiveness and applicability of the framework described above (see Algorithm 1). The examples presented focus on reversible hyperelastic constitutive models, where physical dissipation is not present in the problem.

Three stabilised mixed-based SPH methodologies are analysed, namely $\{\boldsymbol{p}, \boldsymbol{F}\},\{\boldsymbol{p}, \boldsymbol{F}, J\}$ and $\{\boldsymbol{p}, \boldsymbol{F}, \boldsymbol{H}, J\}$ SUPG-SPH, in conjunction with the two strategies described in Section 4.2 for the evaluation of the SUPG stabilising contributions, namely SUPG-SPH-H1 and SUPG-SPH-H6. For comparison purposes, some of the results are benchmarked against the non-LBB compliant B-bar [59] Hexahedral element and the LBB compliant Taylor-Hood Hexahedral element [60], as well as additional in-house mixed-based Finite Element [2-5, 33], Finite Volume [32, 36, 41, 42] and SPH [1] numerical strategies. 


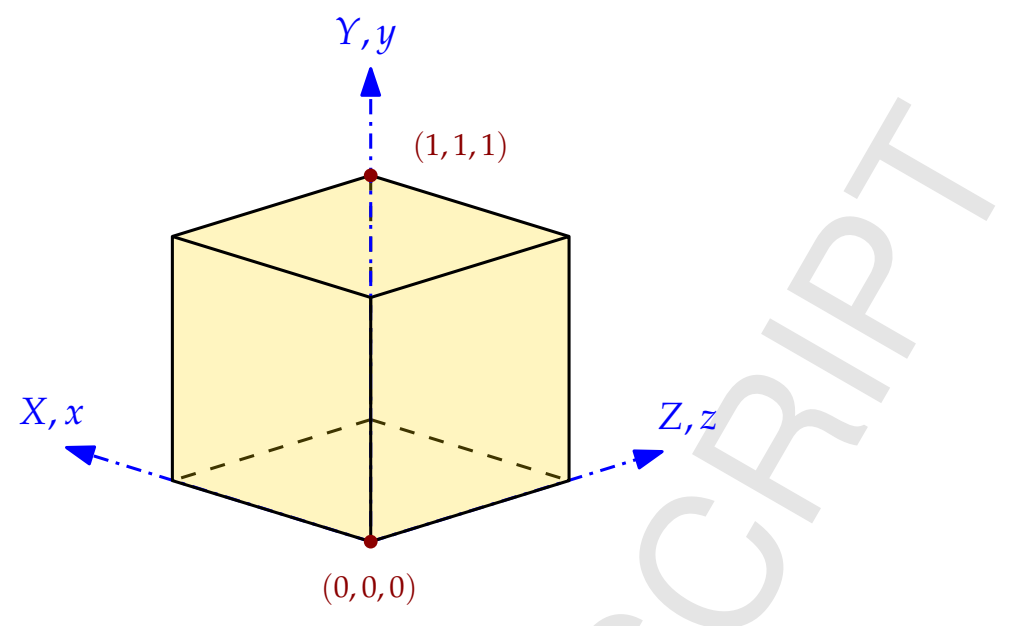

Figure 3: Swinging cube configuration

\subsection{Convergence, consistency and conservation}

In the following section, two different numerical examples are considered in order to assess the performance of the SUPG-SPH algorithm in terms of consistency, convergence and conservation.

The first example shows a swinging cube (see Figure 3 ) of unit side length with symmetric boundary conditions (e.g. restricted normal displacement) at faces $X=0, Y=0$ and $Z=0$ and skew-symmetric boundary conditions (i.e. restricted tangential displacement) at faces $X=1 \mathrm{~m}, Y=1 \mathrm{~m}$ and $Z=1 \mathrm{~m}$ (already presented in References $[2-5,32,36,41,42,47]$ ). The main aim of this example is to show the convergence behaviour of the proposed $\{\boldsymbol{p}, \boldsymbol{F}\}$, $\{\boldsymbol{p}, \boldsymbol{F}, J\}$ and $\{\boldsymbol{p}, \boldsymbol{F}, \boldsymbol{H}, J\}$ SUPG-SPH methodologies in a three dimensional setting. A neo-Hookean material with Young's modulus $E=17 \mathrm{MPa}$ and Poisson's ratio $\nu=0.3$ is considered. The density of the material is $\rho_{0}=1100 \mathrm{~kg} / \mathrm{m}^{3}$ and the Courant-Friedrich-Levy number is $\alpha_{C F L}=0.3$. Following Reference [1] (see Section 8.2 on pg. 85 in Reference [1]), the convergence analysis is carried out by computing the $L_{2}$ norm of the error between the analytical solution of this problem and the numerical solution obtained for different values of the (structured) particle spacing. ${ }^{5}$ Figure 4 shows the expected equal second order convergence pattern for both linear momentum and the components of the stress tensor for all the SUPG-SPH methodologies considered. This shows a clear advantage of the proposed formulations with respect to the classical displacement-based SPH, which yields first order of convergence for stresses. More interestingly, the proposed SUPG-SPH method shows better accuracy than the JST-SPH algorithm previously reported in [1], with the same slope but with a lower translation error (see Figure 5).

In order to assess the ability of the algorithm to preserve angular momentum over a long period of computational time, the L-shaped block example, (see Figure 6), originally proposed by [61] and subsequently studied in References [4, 5, 36, 41, 42], is included. The motion of a three dimensional block subjected to an initial impulse traction at two of its sides (refer to [5], Eqn. (139) in Section 6.6 on pg. 721) is analysed. A neo-Hookean material with Young's modulus $E=50046 \mathrm{~Pa}$ and Poisson's ratio $\nu=0.3$ is considered. The density of the

\footnotetext{
${ }^{5}$ Nearly identical results have been obtained for an unstructured particle distribution, hence not presented.
} 


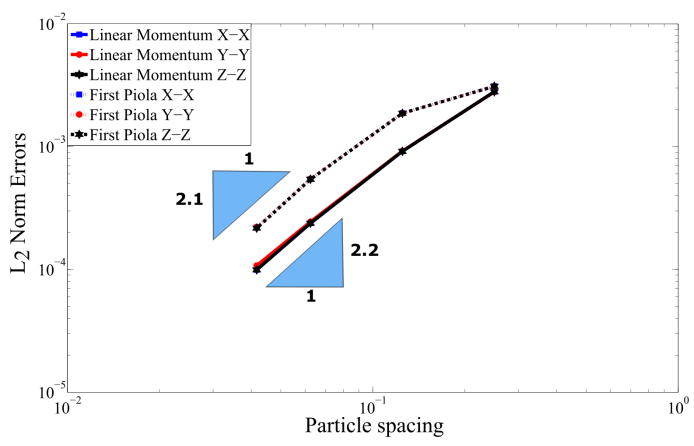

(a) Mixed-based $\{\boldsymbol{p}, \boldsymbol{F}\}$ SUPG-SPH-H1

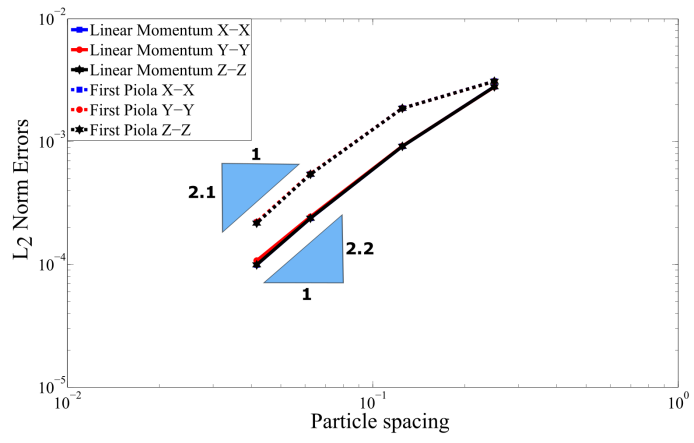

(c) Mixed-based $\{\boldsymbol{p}, \boldsymbol{F}, J\}$ SUPG-SPH-H1

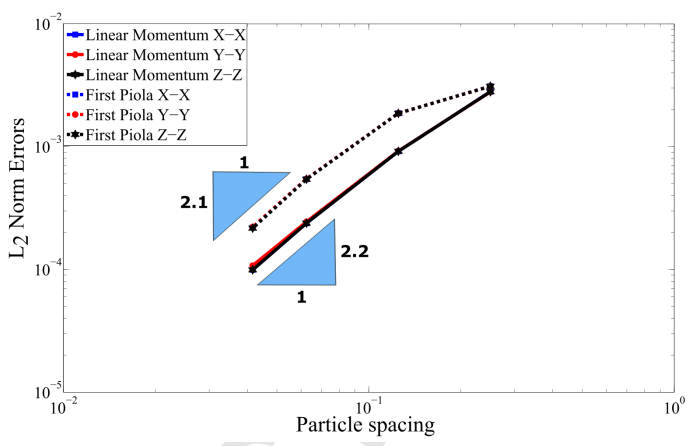

(b) Mixed-based $\{\boldsymbol{p}, \boldsymbol{F}\}$ SUPG-SPH-H6

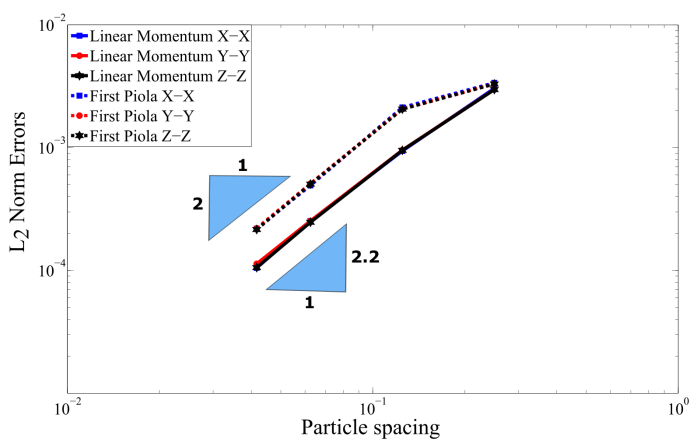

(d) Mixed-based $\{\boldsymbol{p}, \boldsymbol{F}, J\}$ SUPG-SPH-H6

Figure 4: Swinging cube: $L_{2}$ norm convergence of the components of both linear momentum and stresses using the proposed SUPG-SPH methodologies. A neo-Hookean material is used with density $\rho_{0}=1100 \mathrm{~kg} / \mathrm{m}^{3}$, Young's modulus $E=17 \mathrm{MPa}$, Poisson's ratio $\nu=0.3$ and $\alpha_{C F L}=0.3$. SUPG parameters used: SUPG-SPH-H6 $\left\{\tau_{\boldsymbol{F}}=\Delta t, \tau_{\boldsymbol{p}}=\xi_{\boldsymbol{F}}=0\right\}$ and SUPG-SPH-H1 $\left\{\tau_{\boldsymbol{F}}=\Delta t, \xi_{\boldsymbol{F}}=0.1, \tau_{\boldsymbol{p}}=0\right\}$.

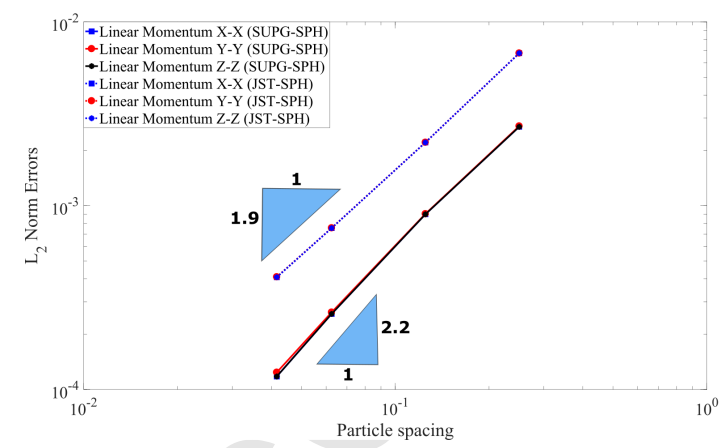

(a) Linear momentum

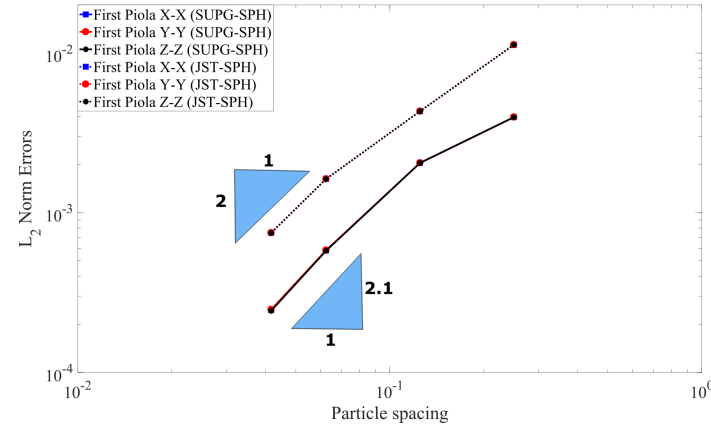

(b) First Piola Kirchhoff stress

Figure 5: Swinging cube: Comparison of $L_{2}$ norm convergence of the components of both linear momentum and stresses using $\{\boldsymbol{p}, \boldsymbol{F}, \boldsymbol{H}, J\}$ SUPG-SPH and $\{\boldsymbol{p}, \boldsymbol{F}, \boldsymbol{H}, J\}$ JST-SPH methodologies. A neo-Hookean material is used with density $\rho_{0}=1100 \mathrm{~kg} / \mathrm{m}^{3}$, Young's modulus $E=17 \mathrm{MPa}$, Poisson's ratio $\nu=0.3$ and $\alpha_{C F L}=0.3$. SUPG-SPH-H1 $\left\{\tau_{\boldsymbol{F}}=\Delta t\right.$, $\left.\xi_{\boldsymbol{F}}=0.1, \tau_{\boldsymbol{p}}=0\right\}$. JST pameters: $\varepsilon_{\boldsymbol{p}}^{(2)}=\varepsilon_{J}^{(2)}=\varepsilon_{J}^{(4)}=0$ and $\varepsilon_{\boldsymbol{p}}^{(4)}=\frac{1}{8}$. 


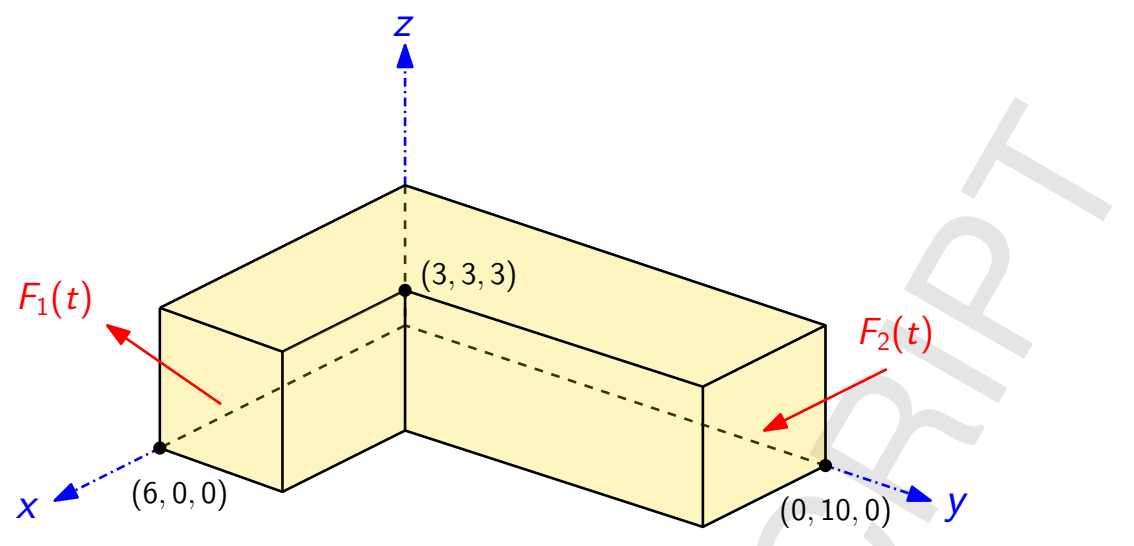

Figure 6: L-shaped block configuration

material is $\rho_{0}=1000 \mathrm{~kg} / \mathrm{m}^{3}$ and the Courant-Friedrich-Levy number is $\alpha_{C F L}=0.3$. Figure 7 illustrates the evolution of the components of both linear and angular momentum for the $\{\boldsymbol{p}, \boldsymbol{F}\}$ SUPG-SPH-H6 formulation using a disordered (secondary) particle arrangement. As it can be observed, the angular momentum of the system remains constant once the external loads are removed whereas linear momentum is zero up to machine accuracy. In addition, a sequence of deformed states is depicted in Figure 8, showing a smooth distribution for the pressure field.

\subsection{Spurious pressure instabilities}

A block of $1 \mathrm{~m} \times 0.5 \mathrm{~m} \times 0.1 \mathrm{~m}$, originally proposed in Reference [58], is left free on its top face and constrained with rollers (i.e. symmetric boundary conditions) on the rest of the boundaries (see Figure 9). The main objective of this example is to show the capability of the algorithm in suppressing spurious pressure oscillations in highly constrained problems. The block is initially compressed with a uniform velocity field $\boldsymbol{v}_{0}=(0,0,-10)^{T} \mathrm{~m} / \mathrm{s}$ applied on a region of the top face, as described in Reference [1] (see Eqn. (54) on pg. 91). A nearly incompressible neo-Hookean material is considered with Young's Modulus $E=1 \mathrm{MPa}$ and Poisson's ratio $\nu=0.499$ (i.e. incompressibility limit $\frac{\kappa}{\mu} \approx 500$ ). The density of the material is $\rho_{0}=1000 \mathrm{~kg} / \mathrm{m}^{3}$.

For benchmarking purposes, the classical displacement-based SPH and JST-SPH method are used. As it is well known, the displacement-based SPH shows excessive pressure fluctuations which eventually leads to an incorrect deformation pattern (see Figure 10a). As it can be observed from Figures (10b) and (10c), severe pressure fluctuations can still be detected using the proposed $\{\boldsymbol{p}, \boldsymbol{F}, \boldsymbol{H}, J\}$ SUPG-SPH if insufficient numerical dissipation $\left(\tau_{\boldsymbol{p}}=0\right)$ is introduced. This spurious pressure mechanism can be entirely eliminated with the inclusion of the appropriate SUPG coefficient $\tau_{\boldsymbol{p}}=0.1 \Delta t$ (see Figure (10d)).

Crucially, for the same number of particles (or degrees of freedom), the variationally consistent SUPG-SPH scheme proves more accurate than the JST-SPH methodology presented in Reference [1]. The latter requires a considerably larger number of particles in order to capture the correct deformation of the block (see Figures (11a) (11b) and (11c)). As it can be observed, both SUPG-SPH-H1 and SUPG-SPH-H6 methodologies show very good agreement in terms of pressure and deformations (see Figures 11c,d), the latter methodology (SUPG-SPH-H6) requiring six times more computational effort for the evaluation of the 


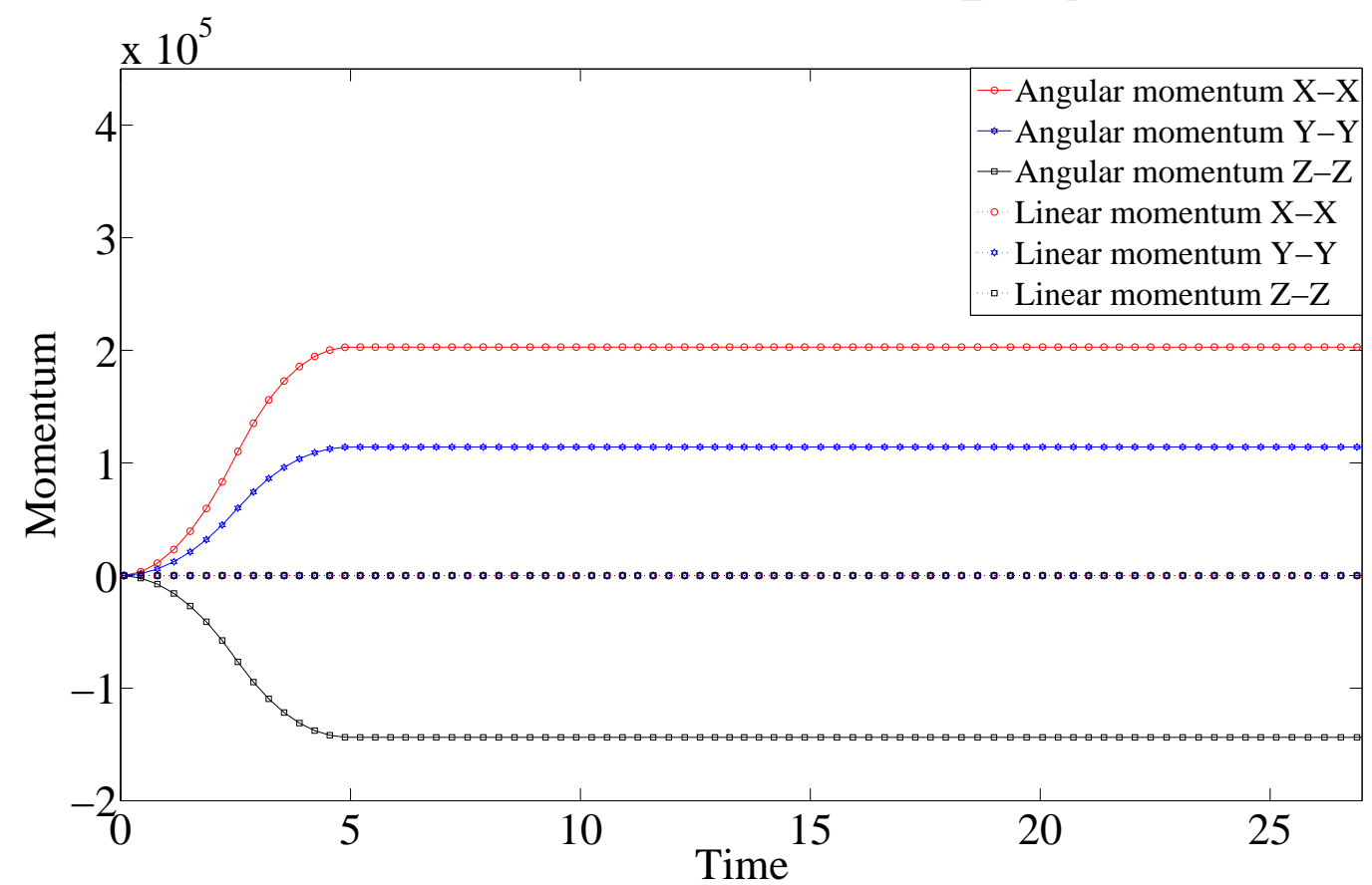

(a)

Figure 7: L-shaped block: Time evolution of the components of angular and linear momentum. Results are obtained using the proposed $\{\boldsymbol{p}, \boldsymbol{F}\}$ SUPG-SPH-H6 with the consideration of the angular momentun projection algorithm. A neo-Hookean material is used with density $\rho_{0}=1000 \mathrm{~kg} / \mathrm{m}^{3}$, Young's modulus $E=50046 \mathrm{~Pa}$, Poisson's ratio $\nu=0.3$ and $\alpha_{C F L}=0.3$. Discretisation of 388 disordered material particles. SUPG parameters used: $\tau_{\boldsymbol{F}}=\Delta t, \xi_{\boldsymbol{F}}=0$. 
$t=2.1424 \mathrm{~s}$

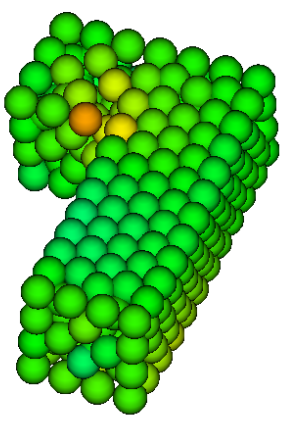

$t=8.1586 \mathrm{~s}$

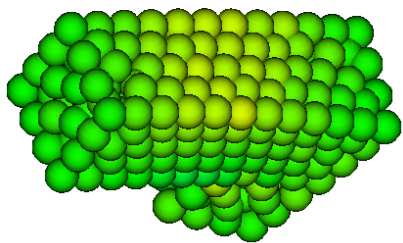

$t=14.143 \mathrm{~s}$

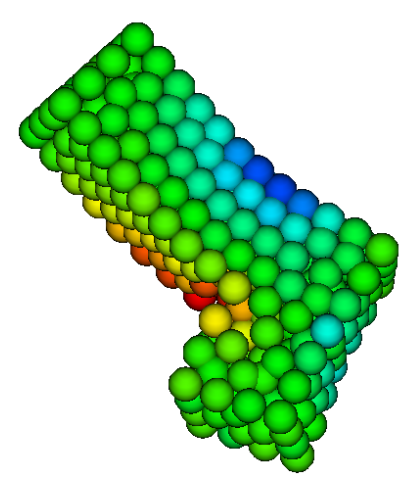

$t=4.1553 \mathrm{~s}$

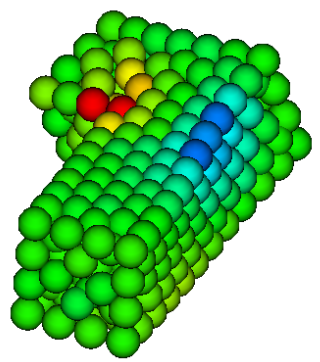

$t=10.1769 \mathrm{~s}$

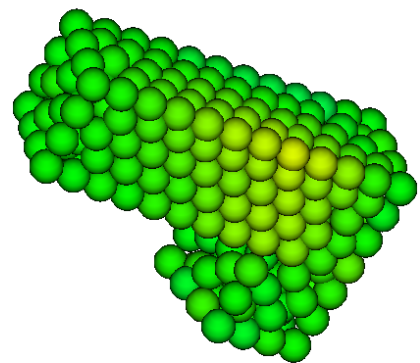

$t=16.2032 \mathrm{~s}$

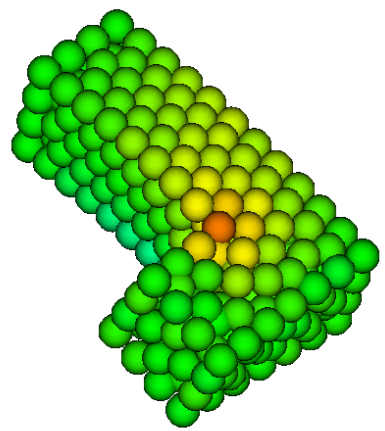

$t=4.1553 \mathrm{~s}$

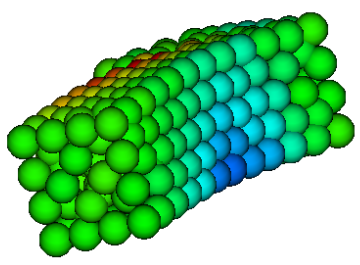

$t=12.2002 \mathrm{~s}$

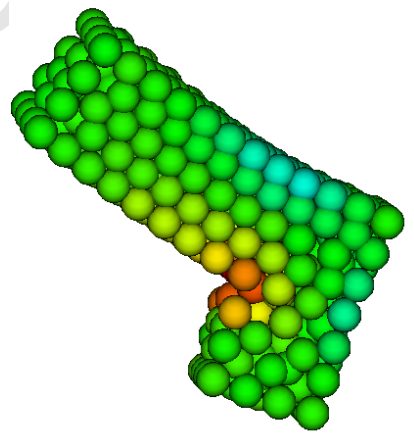

$t=18.2191 \mathrm{~s}$

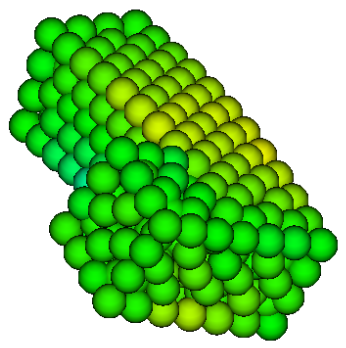

$1100 \quad 2.200 e+03$

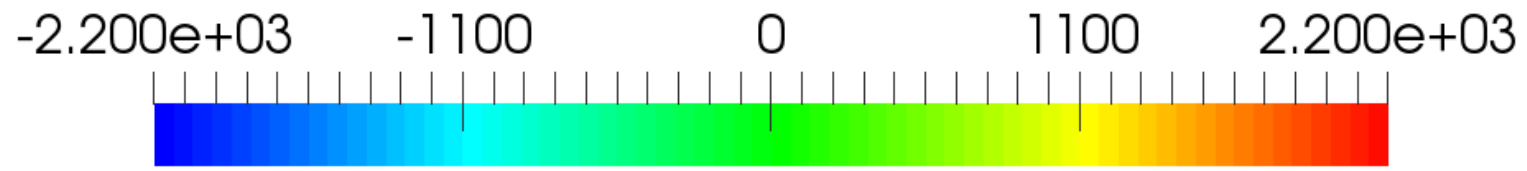

Pressure $(\mathrm{Pa})$

Figure 8: L-shaped block: Time evolution of the deformation plotted with pressure distribution using $\{\boldsymbol{p}, \boldsymbol{F}\}$ SUPG-SPH-H6. A neo-Hookean material is used with density $\rho_{0}=1000$ $\mathrm{kg} / \mathrm{m}^{3}$, Young's modulus $E=50046 \mathrm{~Pa}$, Poisson's ratio $\nu=0.3$ and $\alpha_{C F L}=0.3$. Dicretisation of 388 disordered material particles. SUPG parameters used: $\tau_{\boldsymbol{F}}=\Delta t, \xi_{\boldsymbol{F}}=0$. 


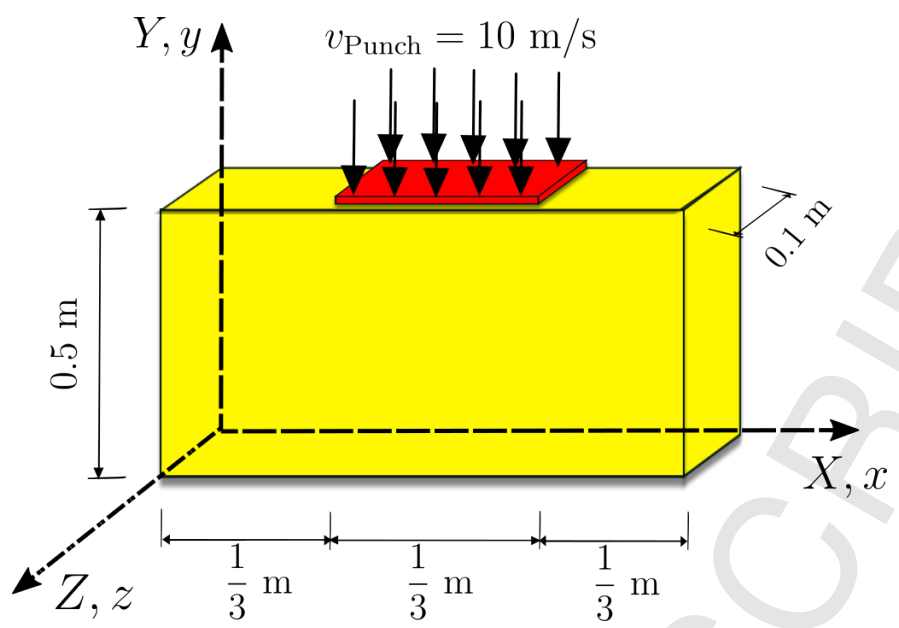

Figure 9: Punch test configuration

SUPG contributions $\mathcal{A}_{\mathrm{SUPG}}^{\{\boldsymbol{p}, J\}}$ in (20) and (29c). It is this reason that leads us to prefer the use of the SUPG-SPH-H1 methodology.

Finally, Figure 12 shows the excellent performance of the mixed-based $\{\boldsymbol{p}, \boldsymbol{F}, \boldsymbol{H}, J\}$ SUPG-SPH-H1 formulation even when extremely large distortions are involved. Notice that in this type of scenarios, mesh-based methods such as the Petrov-Galerkin Finite Element Method (PG-FEM) presented in [2-5] are not suitable unless adaptive mesh refinement is carried out [62].

\subsection{Robustness of the algorithm}

In order to examine the robustness and applicability of the algorithm, a more challenging example (see Figure 13), proposed in References [3-5, 36, 41, 47], is considered in this section. The problem description and material properties are exactly the same as those presented in Section 8.9 on pg. 95 in Reference [1].

The results obtained with three different meshes $(4 \times 19 \times 4,5 \times 25 \times 5$ and $6 \times 31 \times 6)$ using both the mixed-based $\{\boldsymbol{p}, \boldsymbol{F}, \boldsymbol{H}, J\}$ SUPG-SPH-H1 and $\{\boldsymbol{p}, \boldsymbol{F}, \boldsymbol{H}, J\}$ SUPG-SPH-H6 methodologies are shown in Figure 14, displaying both formulations practically identical results. This confirms the convenience of the SUPG-SPH-H1 methodology.

For benchmarking purposes, this problem has been solved using a library of in-house numerical methodologies, namely, JST-SPH [1], PG-FEM [2, 3], Constrained-TOUCH [42], the non-LBB compliant B-bar (P1-Q0) Hexahedral FEM [59] and the LBB compliant TaylorHood (Q2-Q1) Hexahedral FEM [60] (refer to Figure 15).

As shown in the Figure, the results of the proposed $\{\boldsymbol{p}, \boldsymbol{F}, \boldsymbol{H}, J\}$ SUPG-SPH (column (b)) formulation agree very well with those of the other (benchmarking) techniques. In addition, the SUPG-SPH formulation has been run with an ultra-fine discretisation (column (a)) to demonstrate the convergence of the solution. It is interesting to note that the results obtained with the JST-SPH algorithm (column (c)) [1] have been obtained with a slightly finer discretisation than those of the SUPG-SPH formulation in column (b). This is due to the higher numerical dissipation of the JST method [36, 42].

It is worth emphasising the accuracy of the results obtained, comparable to those of the PG-FEM formulation presented in [2-5]. In the latter, a VMS stabilisation strategy 
$t=0.005 \mathrm{~s}$

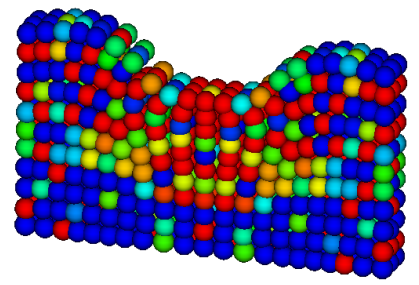

(a) Displacement-based formulation

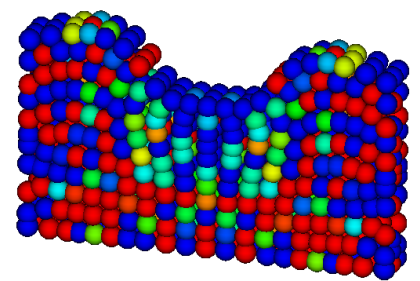

$t=0.015 \mathrm{~s}$
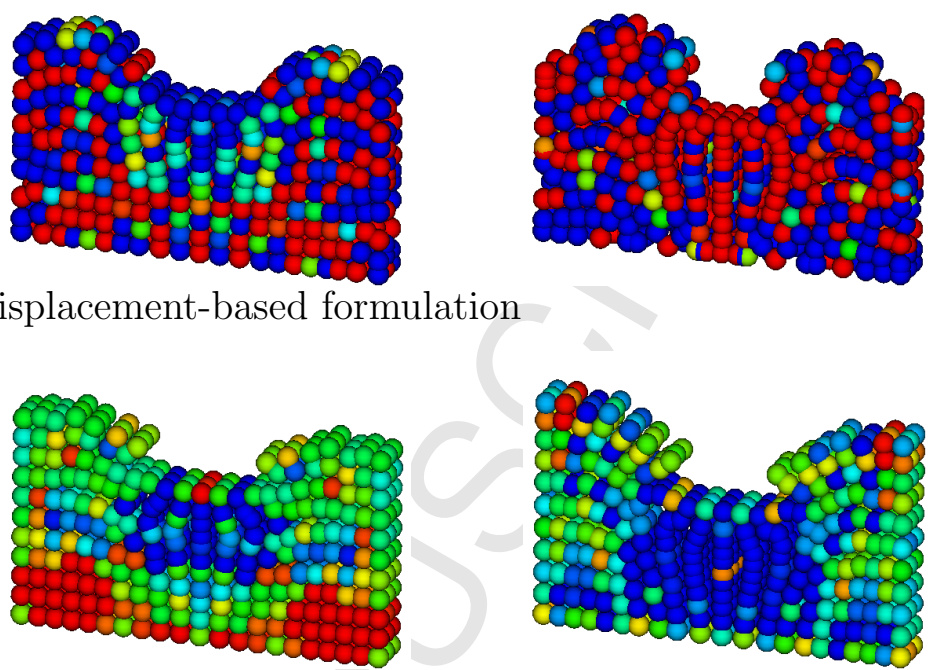

(b) Mixed-based $\{\boldsymbol{p}, \boldsymbol{F}, \boldsymbol{H}, J\} \mathrm{SPH}\left(\tau_{\boldsymbol{F}}=\tau_{\boldsymbol{p}}=\xi_{\boldsymbol{F}}=0\right)$
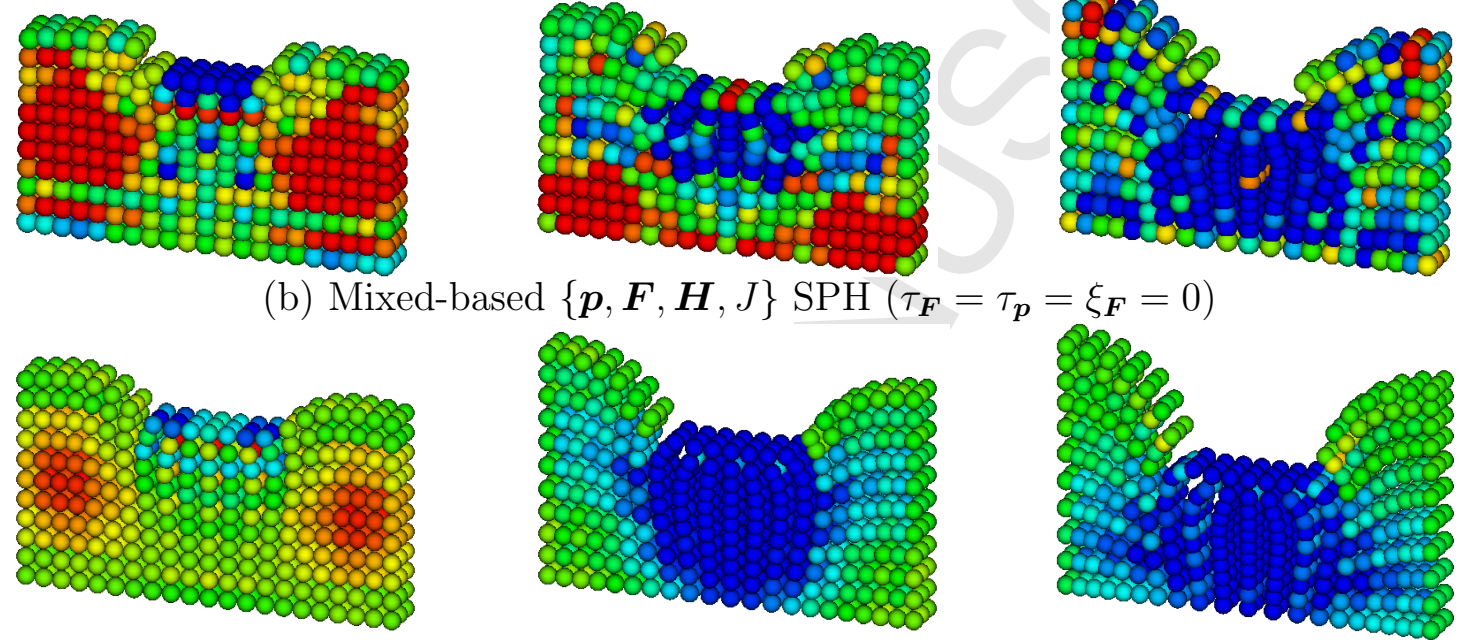

(c) Mixed-based $\{\boldsymbol{p}, \boldsymbol{F}, \boldsymbol{H}, J\}$ SUPG-SPH-H6 $\left(\tau_{\boldsymbol{F}}=0.5 \Delta t, \tau_{\boldsymbol{p}}=\xi_{\boldsymbol{F}}=0\right)$
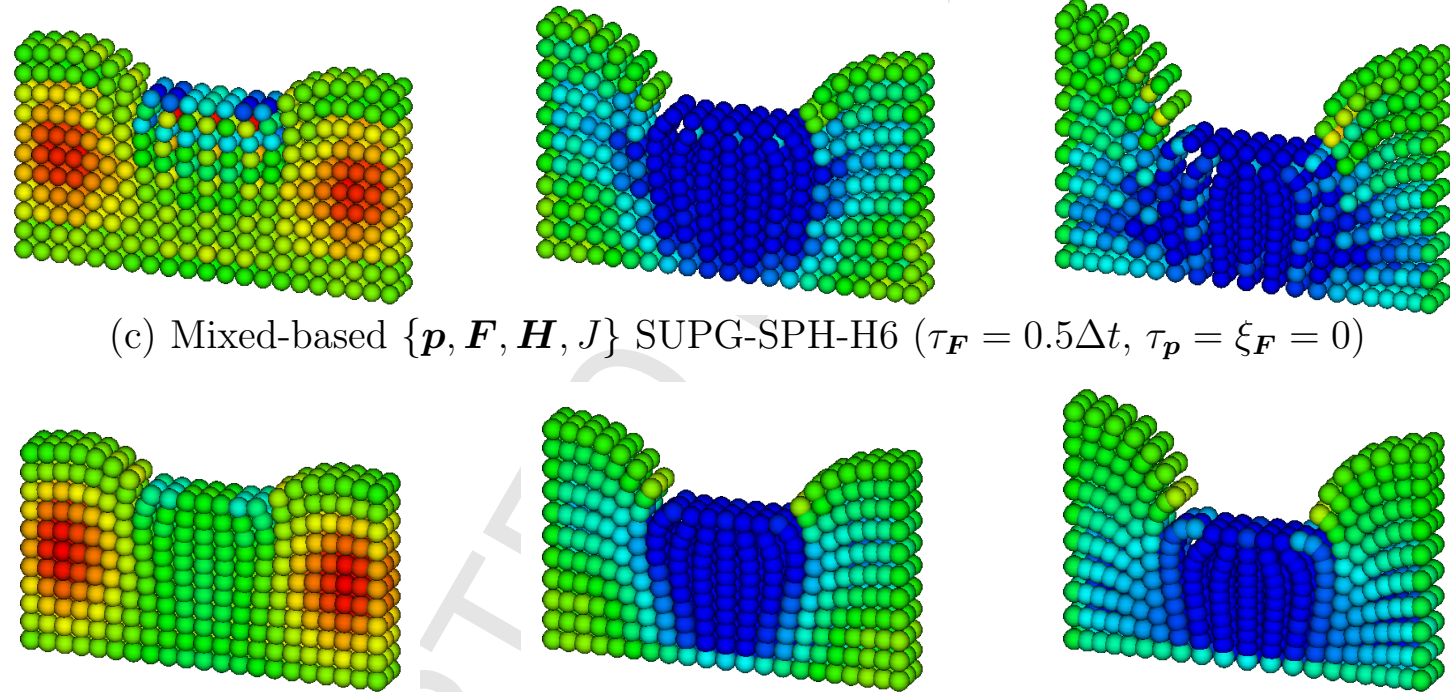

(d) Mixed-based $\{\boldsymbol{p}, \boldsymbol{F}, \boldsymbol{H}, J\}$ SUPG-SPH-H6 $\left(\tau_{\boldsymbol{F}}=0.5 \Delta t, \tau_{\boldsymbol{p}}=0.1 \Delta t, \xi_{\boldsymbol{F}}=0\right)$

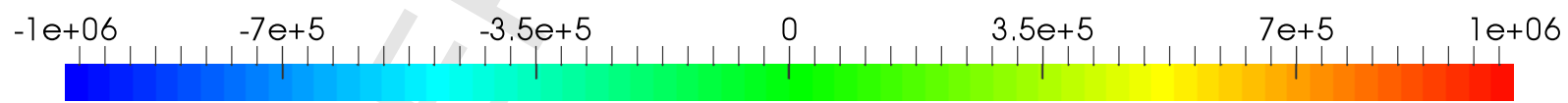

\section{Pressure $(\mathrm{Pa})$}

Figure 10: Nearly incompressible punch test: Time evolution of the deformation plotted with pressure distribution using (a) Displacement-based formulation; (b) $\boldsymbol{p}$ - $\boldsymbol{F}-\boldsymbol{H}$ - $J$ SPH $\left(\tau_{\boldsymbol{F}}=\tau_{\boldsymbol{p}}=\xi_{\boldsymbol{F}}=0\right)$; (c) $\boldsymbol{p}-\boldsymbol{F}-\boldsymbol{H}-J$ SUPG-SPH-H6 $\left(\tau_{\boldsymbol{F}}=0.5 \Delta t, \tau_{\boldsymbol{p}}=\xi_{\boldsymbol{F}}=0\right)$; and (d) $\boldsymbol{p}$ - $\boldsymbol{F}-\boldsymbol{H}-J$ SUPG-SPH-H6 $\left(\tau_{\boldsymbol{F}}=0.5 \Delta t, \tau_{\boldsymbol{p}}=0.1 \Delta t\right.$ and $\left.\xi_{\boldsymbol{F}}=0\right)$. A neo-Hookean material is used with density $\rho_{0}=1000 \mathrm{~kg} / \mathrm{m}^{3}$, Young's modulus $E=1 \mathrm{MPa}$, Poisson's ratio $\nu=0.499$ and $\alpha_{C F L}=0.3$. 


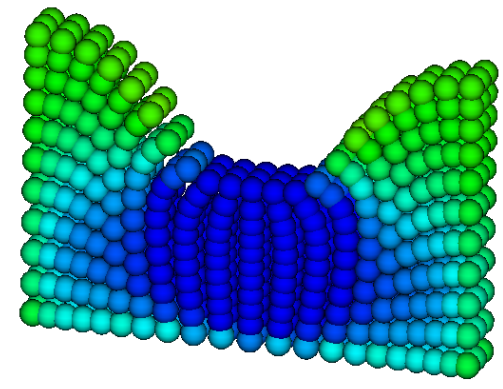

(a)

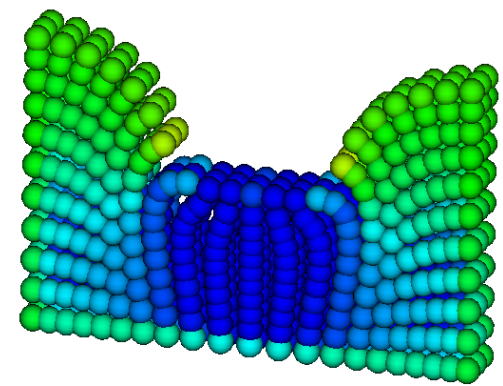

(c)

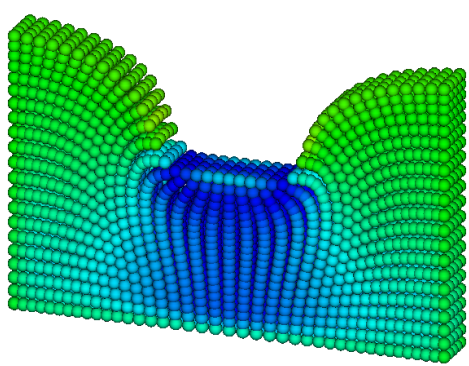

(b)

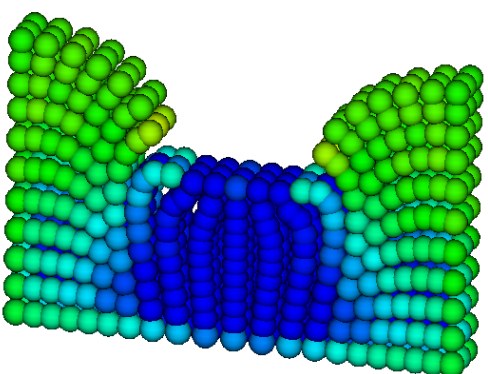

(d)

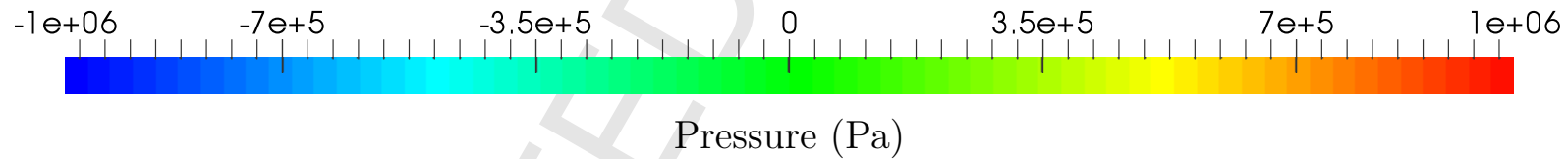

Figure 11: Nearly incompressible punch test: Comparison of deformation plotted with pressure distribution at time $t=0.015$ s using: (a) Mixed-based $\{\boldsymbol{p}, \boldsymbol{F}, \boldsymbol{H}, J\}$ JST-SPH $(21 \times 11 \times 3$ particles $)$; (b) Mixed-based $\{\boldsymbol{p}, \boldsymbol{F}, \boldsymbol{H}, J\}$ JST-SPH $(41 \times 21 \times 4$ particles $)$; (c) Mixed-based $\{\boldsymbol{p}, \boldsymbol{F}, \boldsymbol{H}, J\}$ SUPG-SPH-H6 $\left(21 \times 11 \times 3\right.$ particles with $\tau_{\boldsymbol{F}}=0.5 \Delta t ; \tau_{\boldsymbol{p}}=0.1 \Delta t$; $\left.\xi_{\boldsymbol{F}}=0\right)$; and (d) Mixed-based $\{\boldsymbol{p}, \boldsymbol{F}, \boldsymbol{H}, J\}$ SUPG-SPH-H1 $(21 \times 11 \times 3$ particles with $\left.\tau_{\boldsymbol{F}}=0.5 \Delta t ; \tau_{\boldsymbol{p}}=0.1 \Delta t ; \xi_{\boldsymbol{F}}=0.1\right)$. A neo-Hookean material is used with density $\rho_{0}=1000$ $\mathrm{kg} / \mathrm{m}^{3}$, Young's modulus $E=1 \mathrm{MPa}$, Poisson's ratio $\nu=0.499$ and $\alpha_{C F L}=0.3$. JST parameters: $\varepsilon_{\boldsymbol{p}}^{(2)}=\varepsilon_{J}^{(2)}=0, \varepsilon_{\boldsymbol{p}}^{(4)}=\frac{1}{8}, \varepsilon_{J}^{(4)}=\frac{1}{32}$. 
$t=0 \mathrm{~s}$

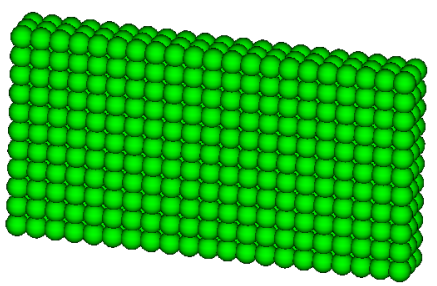

$t=0.0271 \mathrm{~s}$

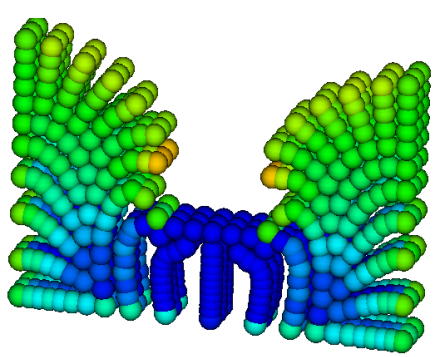

$t=0.0378 \mathrm{~s}$

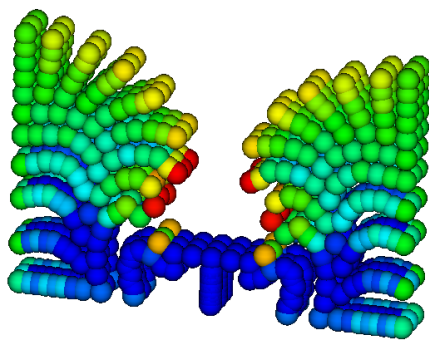

$t=0.0118 \mathrm{~s}$

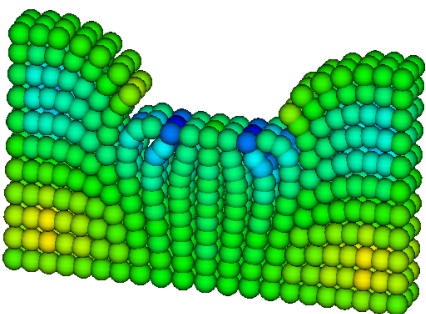

$t=0.0318 \mathrm{~s}$

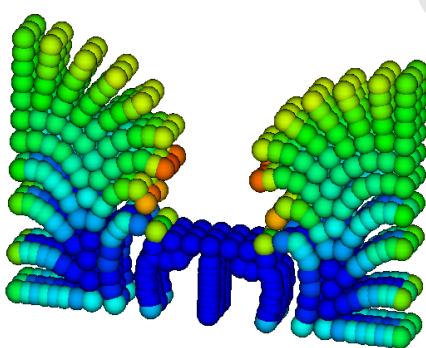

$t=0.0394 \mathrm{~s}$

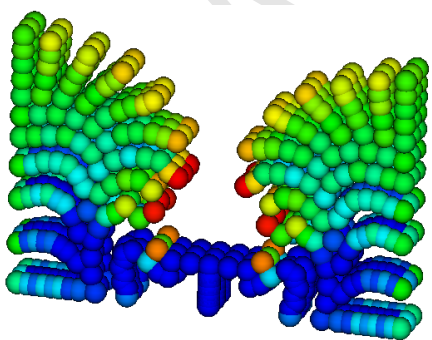

$t=0.0203 \mathrm{~s}$

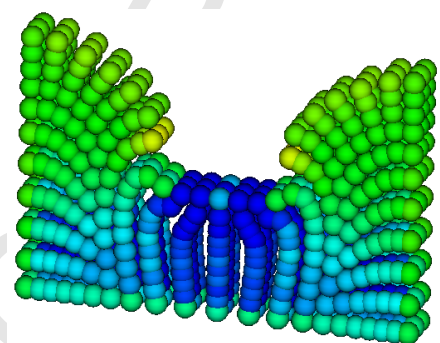

$t=0.0353 \mathrm{~s}$

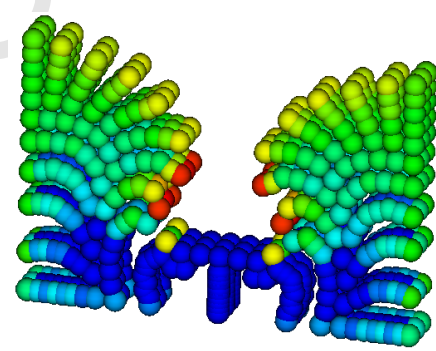

$t=0.0403 \mathrm{~s}$

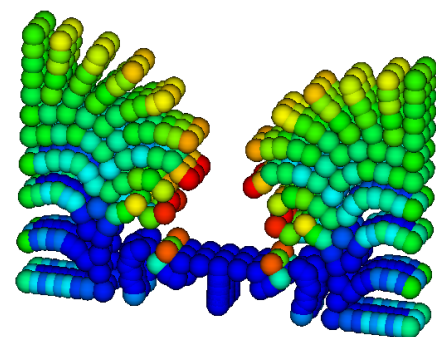

$-1 e+06 \quad-7 e+5 \quad-3.5 e+5 \quad 0 \quad 3.5 e+5 \quad 7 e+5 \quad 1 e+06$

Pressure $(\mathrm{Pa})$

Figure 12: Nearly incompressible punch test: A sequence of deformed states plotted with pressure distribution using the mixed-based $\{\boldsymbol{p}, \boldsymbol{F}, \boldsymbol{H}, J\}$ SUPG-SPH-H1. A neo-Hookean material is used with density $\rho_{0}=1000 \mathrm{~kg} / \mathrm{m}^{3}$, Young's modulus $E=1 \mathrm{MPa}$, Poisson's ratio $\nu=0.499$ and $\alpha_{C F L}=0.3$. Discretisation of $21 \times 11 \times 3$ material particles. SUPG parameters used: $\tau_{\boldsymbol{F}}=0.5 \Delta t, \tau_{\boldsymbol{p}}=0.1 \Delta t, \xi_{\boldsymbol{F}}=0.1$. 


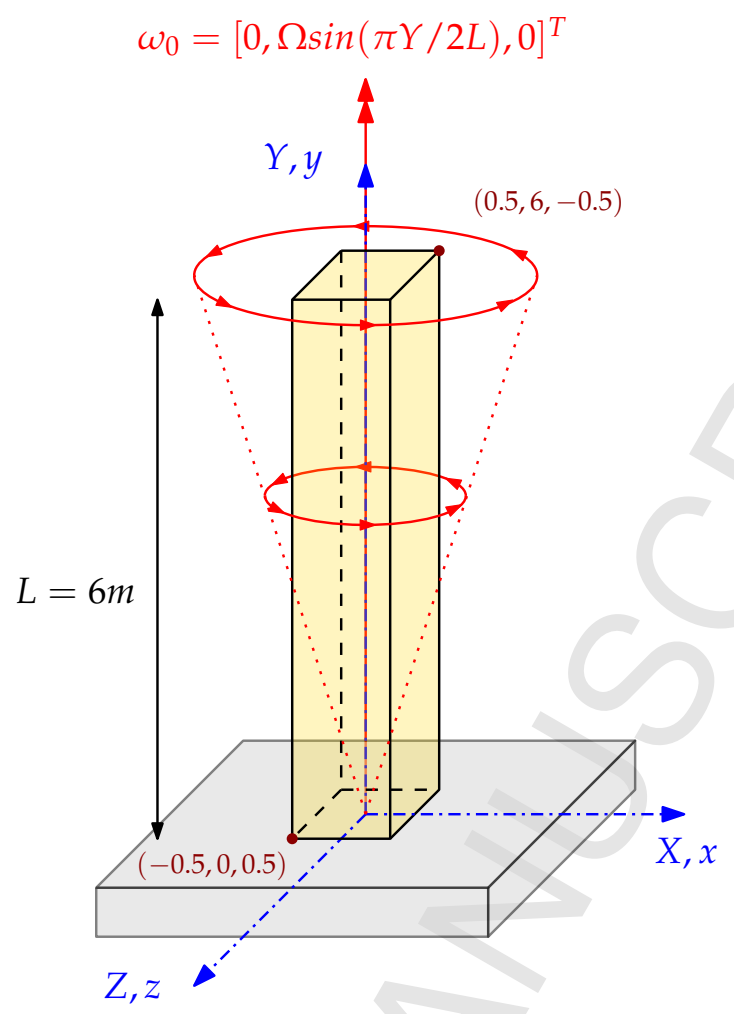

Figure 13: Twisting column configuration

was followed, requiring a higher number of stabilisation parameters than in the SUPG-SPH framework proposed in this paper (refer to Section 3.1).

\section{Conclusions}

This paper presents a novel SPH computational framework tailor-made for the system of first order conservation laws $\{\boldsymbol{p}, \boldsymbol{F}, \boldsymbol{H}, J\}$ introduced in $[4,5]$ in the context of explicit fast solid dynamics. The proposed methodology explores a new SUPG stabilisation technique displaying a series of advantages over the recently proposed JST-SPH framework in [1]. First, the variationally consistent nature of the SUPG stabilisation allows for the introduction of a locally preserving angular momentum procedure. This differs from the JST-SPH framework, where an a posteriori projection procedure was required to ensure global angular momentum preservation. Second, evaluation of expensive harmonic and bi-harmonic operators, necessary for the JST stabilisation, is avoided in the new SUPG-SPH framework. Third, the SUPG-SPH framework is more accurate (for the same number of degrees of freedom) than its JST-SPH counterpart and its accuracy is comparable to that of the robust (but computationally more demanding) Petrov Galerkin Finite Element Method (PG-FEM) technique explored by the authors in [2-5], which requires a higher number of stabilisation parameters. The latter formulation, being mesh-based, cannot handle problems involving large distorsions, as opposed to the formulation developed. These advantages indicate the potential and applicability of the proposed formulation in real large scale simulations.

In forthcoming publications, the authors will further explore the formulation proposed studying three new aspects: first, the use of an entropy-based formulation for nearly and truly 
$4 \times 19 \times 4$
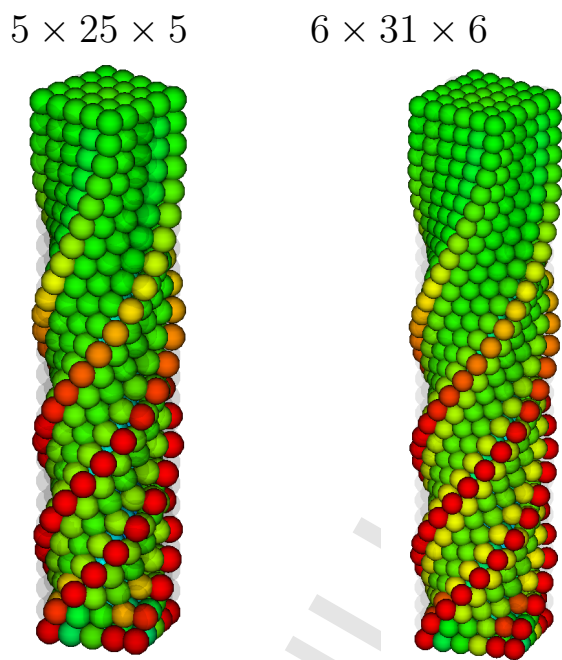

(a) Mixed-based $\{\boldsymbol{p}, \boldsymbol{F}, \boldsymbol{H}, J\}$ SUPG-SPH-H6
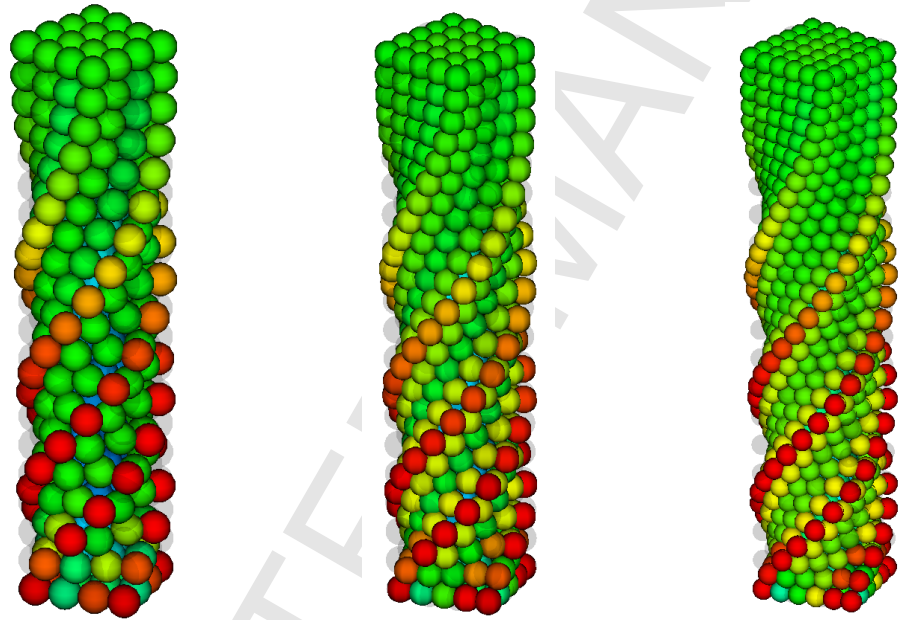

(b) Mixed-based $\{\boldsymbol{p}, \boldsymbol{F}, \boldsymbol{H}, J\}$ SUPG-SPH-H1

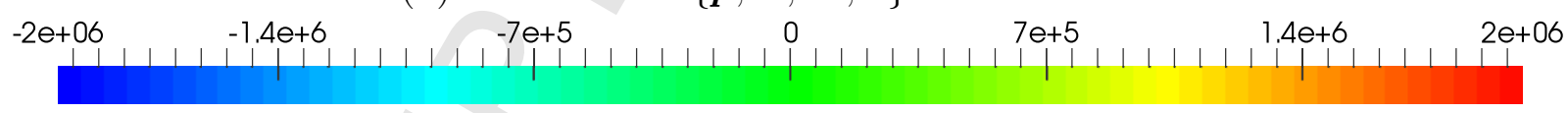

\section{Pressure $(\mathrm{Pa})$}

Figure 14: Twisting column: A sequence of particle refinement analysis at a particular time $t=0.1$ s using: (a) Mixed-based $\{\boldsymbol{p}, \boldsymbol{F}, \boldsymbol{H}, J\}$ SUPG-SPH-H6 $\left(\tau_{\boldsymbol{F}}=\Delta t, \xi_{\boldsymbol{F}}=0, \tau_{\boldsymbol{p}}=0.1 \Delta t\right)$; and (b) Mixed-based $\{\boldsymbol{p}, \boldsymbol{F}, \boldsymbol{H}, J\}$ SUPG-SPH-H1 $\left(\tau_{\boldsymbol{F}}=\Delta t, \xi_{\boldsymbol{F}}=0.2, \tau_{\boldsymbol{p}}=0.1 \Delta t\right)$. Results obtained with an angular velocity field $\boldsymbol{\omega}_{0}=[0, \Omega \sin (\pi Y / 2 L), 0]$ where $\Omega=105 \mathrm{rad} / \mathrm{s}$ and $L=6 \mathrm{~m}$. A neo-Hookean material is used with density $\rho_{0}=1100 \mathrm{~kg} / \mathrm{m}^{3}$, Young's modulus $E=17 \mathrm{MPa}$, Poisson's ratio $\nu=0.495$ and $\alpha_{C F L}=0.3$. 


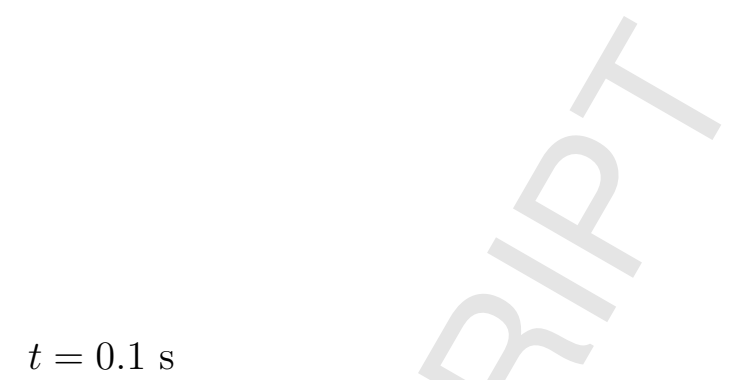

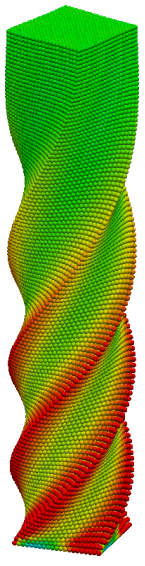

(a)

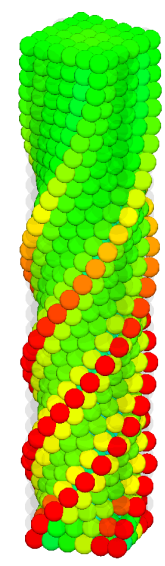

(b)

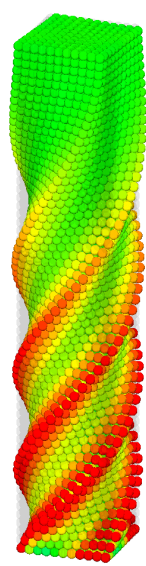

(c)

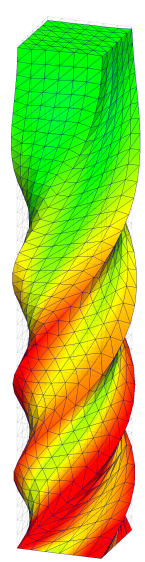

(d)

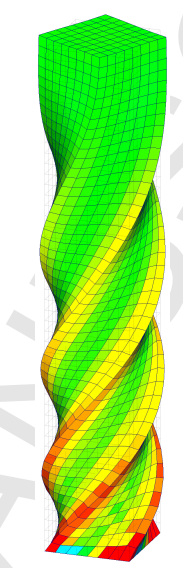

(e)

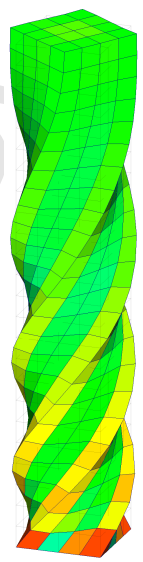

(f)

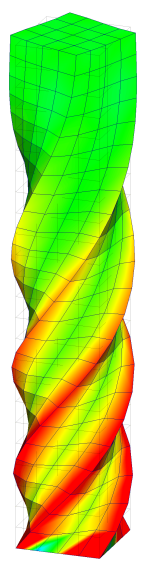

(g)

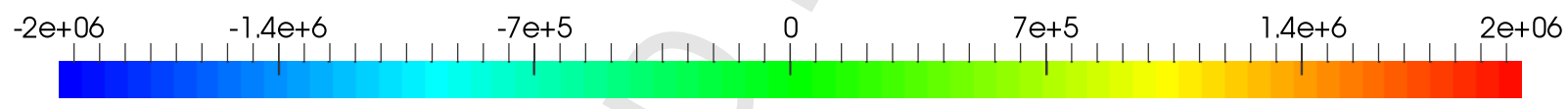

\section{Pressure $(\mathrm{Pa})$}

Figure 15: Twisting column: Comparison of deformed shapes plotted with pressures at time $t=0.1$ s using: (a) Mixed-based $\{\boldsymbol{p}, \boldsymbol{F}, \boldsymbol{H}, J\}$ SUPG-SPH-H1 with ultra-fine discretisation $\left(\tau_{\boldsymbol{F}}=\Delta t, \xi_{\boldsymbol{F}}=0.2, \tau_{\boldsymbol{p}}=0.1 \Delta t\right) ;(\mathrm{b})$ Mixed-based $\{\boldsymbol{p}, \boldsymbol{F}, \boldsymbol{H}, J\}$ SUPG-SPH-H1 $\left(\tau_{\boldsymbol{F}}=\right.$ $\left.\Delta t, \xi_{\boldsymbol{F}}=0.2, \tau_{\boldsymbol{p}}=0.1 \Delta t\right)$; (c) Mixed-based $\{\boldsymbol{p}, \boldsymbol{F}\}$ JST-SPH $\left(\varepsilon_{\boldsymbol{p}}^{(2)}=0\right.$ and $\left.\varepsilon_{\boldsymbol{p}}^{(4)}=\frac{1}{8}\right)$; (d) PG-FEM [3]; (e) Constrained-TOUCH [42]; (f) B-bar hexahedral method [59]; and (g) Taylor-Hood (Q2-Q1) hexahedral FEM [60]. Results obtained with an angular velocity field $\boldsymbol{\omega}_{0}=[0, \Omega \sin (\pi Y / 2 L), 0]$ where $\Omega=105 \mathrm{rad} / \mathrm{s}$ and $L=6 \mathrm{~m}$. A neo-Hookean material is used with density $\rho_{0}=1100 \mathrm{~kg} / \mathrm{m}^{3}$, Young's modulus $E=17 \mathrm{MPa}$ and Poisson's ratio $\nu=0.495$. 
incompressible elasticity [3, 4]; second, the consideration of thermoelasticity [41]; and third, the adaptation of the current framework to high strain dynamic fracture and fragmentation with particle refinement [63].

\section{Acknowledgements}

The authors gratefully acknowledge the financial support provided by the Sêr Cymru National Research Network for Advanced Engineering and Materials, United Kingdom. The third author would also like to acknowledge the financial support received through "The Erasmus Mundus Joint Doctorate SEED" programme.

[1] C. H. Lee, A. J. Gil, G. Greto, S. Kulasegaram, J. Bonet, A new Jameson-SchmidtTurkel Smooth Particle Hdrodynamics algorithm for large strain explicit fast dynamics, Computer Methods in Applied Mechanics and Engineering 311 (2016) 71-111.

[2] C. H. Lee, A. J. Gil, J. Bonet, Development of a stabilised Petrov-Galerkin formulation for conservation laws in Lagrangian fast solid dynamics, Computer Methods in Applied Mechanics and Engineering 268 (2014) 40-64.

[3] A. J. Gil, C. H. Lee, J. Bonet, M. Aguirre, A stabilised Petrov-Galerkin formulation for linear tetrahedral elements in compressible, nearly incompressible and truly incompressible fast dynamics, Computer Methods in Applied Mechanics and Engineering 276 (2014) 659-690.

[4] A. J. Gil, C. H. Lee, J. Bonet, R. Ortigosa, A first order hyperbolic framework for large strain computational solid dynamics. Part II: Total Lagrangian compressible, nearly incompressible and truly incompressible elasticity, Computer Methods in Applied Mechanics and Engineering 300 (2016) 146-181.

[5] J. Bonet, A. J. Gil, C. H. Lee, M. Aguirre, R. Ortigosa, A first order hyperbolic framework for large strain computational solid dynamics. Part I: Total Lagrangian isothermal elasticity, Computer Methods in Applied Mechanics and Engineering 283 (2015) 689732 .

[6] P. W. Randles, L. D. Libersky, Smoothed Particle Hydrodynamics: Some recent improvements and applications, Computer Methods in Applied Mechanics and Engineering 139 (1996) 375-408.

[7] G. R. Johnson, S. R. Beissel, Normalized smoothing functions for SPH impact computations, International Journal for Numerical Methods in Engineering 39 (1996) 2725-2741.

[8] Y. Vidal, J. Bonet, A. Huerta, Stabilized updated Lagrangian corrected SPH for explicit dynamic problems, International Journal for Numerical Methods in Engineering 69 (2006) 2687-2710.

[9] J. Bonet, S. Kulasegaram, Remarks on tension instability of Eulerian and Lagrangian Corrected Smooth Particle Hydrodynamics (CSPH) methods, International Journal for Numerical Methods in Engineering 52 (2001) 1203-1220.

[10] G. C. Ganzenmüller, An hourglass control algorithm for Lagrangian Smooth Particle Hydrodynamics, Computer Methods in Applied Mechanics and Engineering 286 (2015) $87-106$. 
[11] G. C. Ganzenmüller, S. Hiermaier, M. May, On the similarity of meshless discretizations of Peridynamics and Smooth-Particle Hydrodynamics, Computers and Structures 150 (2015) 71-78.

[12] S. A. Silling, Reformulation of elasticity theory for discontinuities and long-range forces, Journal of the Mechanics and Physics of Solids 48 (2000) 175-209.

[13] J. W. Swegle, D. L. Hicks, S. W. Attaway, Smoothed Particle Hydrodynamics stability analysis, Journal of Computational Physics 116 (1995) 123-134.

[14] T. Belytschko, Y. Guo, W. K. Liu, S. P. Xiao, A unified stability analysis of meshless particle methods, International Journal for Numerical Methods in Engineering 48 (2000) $1359-1400$.

[15] J. J. Monaghan, SPH without a tensile instability, Journal of Computational Physics 159 (2000) 290-311.

[16] M. A. Puso, J. S. Chen, E. Zywicz, W. Elmer, Meshfree and finite element nodal integration methods, International Journal for Numerical Methods in Engineering 74 (2008) 416-446.

[17] J. Bonet, A. J. Burton, A simple average nodal pressure tetrahedral element for incompressible and nearly incompressible dynamic explicit applications, Communications in Numerical Methods in Engineering 14 (1998) 437-449.

[18] M. W. Gee, C. R. Dohrmann, S. W. Key, W. A. Wall, A uniform nodal strain tetrahedron with isochoric stabilization, International Journal for Numerical Methods in Engineering 78 (2009) 429-443.

[19] C. R. Dohrmann, M. W. Heinstein, J. Jung, S. W. Key, W. R. Witkowski, Node-based uniform strain elements for three-node triangular and four-node tetrahedral meshes, International Journal for Numerical Methods in Engineering 47 (2000) 1549-1568.

[20] M. A. Puso, J. Solberg, A stabilized nodally integrated tetrahedral, International Journal for Numerical Methods in Engineering 67 (2006) 841-867.

[21] M. B. Liu, G. R. Liu, K. Y. Lam, Constructing smoothing functions in smoothed particle hydrodynamics with applications, Jounal of Computational and Applied Mathematics 155 (2003) 263-284.

[22] J. K. Chen, J. E. Beraun, T. C. Carney, A corrective smoothed particle method for boundary value problems in heat conduction, International Journal for Numerical Methods in Engineering 46 (1999) 231-252.

[23] J. Bonet, S. Kulasegaram, Correction and stabilization of smooth particle hydrodynamics methods with applications in metal forming simulations, International Journal for Numerical Methods in Engineering 47 (2000) 1189-1214.

[24] J. J. Monaghan, Smoothed particle hydrodynamics, Annual Review of Astronomy and Astrophysics 30 (1992) 543-574. 
[25] P. W. Randles, L. D. Libersky, Normalized SPH with stress points, International Journal for Numerical Methods in Engineering 48 (2000) 1445-1462.

[26] J. S. Chen, C. T. Wu, S. Yoon, Y. You, A stabilized conforming nodal integration for Galerkin mesh-free methods, International Journal for Numerical Methods in Engineering 50 (2001) 435-466.

[27] T. Blanc, M. Pastor, A stabilized Smoothed Particle Hydrodynamics, Taylor-Galerkin algorithm for soil dynamics problems, International Journal for Numerical and Analytical Methods in Engineering 37 (2013) 1-30.

[28] M. I. Herreros, M. Mabssout, A two-steps time discretization scheme using the SPH method for shock wave propogation, Computer Methods in Applied Mechanics and Engineering 200 (2011) 1833-1845.

[29] M. Mabssout, M. I. Herreros, Runge-Kutta vs Taylor-SPH: Two time integration schemes for SPH with application to soil dynamics, Applied Mathematical Modelling 37 (2013) 3541-3563.

[30] A. Jameson, W. Schmidt, E. Turkel, Numerical solution of the Euler equations by finite volume methods using Runge Kutta time stepping schemes, AIAA 14th Fluid and Plasma Dynamic Conference 81 (1981).

[31] T. J. R. Hughes, L. P. Franca, M. Mallet, A new finite element formulation for computational fluid dynamics: I. Symmetric forms of the compressible Euler and Navier-Stokes equations and the second law of thermodynamics, Computer Methods in Applied Mechanics and Engineering 54 (1986) 223-234.

[32] C. H. Lee, A. J. Gil, J. Bonet, Development of a cell centred upwind finite volume algorithm for a new conservation law formulation in structural dynamics, Computers and Structures 118 (2013) 13-38.

[33] I. A. Karim, C. H. Lee, A. J. Gil, J. Bonet, A two-step Taylor Galerkin formulation for fast dynamics, Engineering Computations 31 (2014) 366-387.

[34] G. Kluth, B. Després, Discretization of hyperelasticity on unstructured mesh with a cell-centered Lagrangian scheme, Journal of Computational Physics 229 (2010) 90929118.

[35] N. C. Nguyen, J. Peraire, Hybridizable Discontinuous Galerkin methods for partial differential equations in continuum mechanics, Journal of Computational Physics 231 (2012) 5955-5988.

[36] M. Aguirre, A. J. Gil, J. Bonet, A. A. Carreño, A vertex centred finite volume JamesonSchmidt-Turkel (JST) algorithm for a mixed conservation formulation in solid dynamics, Journal of Computational Physics 259 (2014) 672-699.

[37] J. Bonet, A. J. Gil, R. Ortigosa, On a tensor cross product based formulation of large strain solid mechanics, International Journal of Solids and Structures 84 (2016) 49-63. 
[38] J. Bonet, A. J. Gil, R. Ortigosa, A computational framework for polyconvex large strain elasticity, Computer Methods in Applied Mechanics and Engineering 283 (2015) 1061-1094.

[39] J. A. Trangenstein, P. Colella, A higher-order Godunov method for modelling finite deformation in elastic-plastic solids, Communications on Pure and Applied Mathematics 44 (1991) 41-100.

[40] J. Bonet, A. J. Gil, R. D. Wood, Nonlinear Solid Mechanics for Finite Element Analysis: Statics, Cambridge University Press, 2016.

[41] M. Aguirre, A. J. Gil, J. Bonet, C. H. Lee, An upwind vertex centred finite volume solver for Lagrangian solid dynamics, Journal of Computational Physics 300 (2015) 387-422.

[42] J. Haider, C. H. Lee, A. J. Gil, J. Bonet, A first order hyperbolic framework for large strain computational solid dynamics: An upwind cell centred Total Lagrangian scheme, International Journal for Numerical Methods in Engineering 109 (2017) 407-456.

[43] T. J. R. Hughes, M. Mallet, A new finite element formulation for computational fluid dynamics: III. The generalized streamline operator for multidimensional advective-diffusive systems, Computer Methods in Applied Mechanics and Engineering 58 (1986) 305-328.

[44] A. N. Brooks, T. J. R. Hughes, Streamline upwind/Petrov-Galerkin formulations for convection dominated flows with particular emphasis on the incompressible Navier-Stokes equations, Computer Methods in Applied Mechanics and Engineering 32 (1982) 199-259.

[45] T. J. R. Hughes, Multiscale phenomena: Green's functions, the Dirichlet-to-Neumann formulation, subgrid scale models, bubbles and the origins of stabilized methods, Computer Methods in Applied Mechanics and Engineering 127 (1995) 387-401.

[46] G. Scovazzi, Lagrangian shock hydrodynamics on tetrahedral meshes: A stable and accurate variational multiscale approach, Journal of Computational Physics 231 (2012) 8029-8069.

[47] G. Scovazzi, B. Carnes, X. Zeng, S. Rossi, A simple, stable, and accurate linear tetrahedral finite element for transient, nearly and fully incompressible solid dynamics: A dynamic variational multiscale approach, International Journal for Numerical Methods in Engineering 106 (2016) 799-839.

[48] S. Rossi, N. Abboud, G. Scovazzi, Implicit finite incompressible elastodynamics with linear finite elements: A stabilized method in rate form, Computer Methods in Applied Mechanics and Engineering 311 (2016) 208-249.

[49] J. Bonet, T.-S. Lok, Variational and momentum preservation aspects of smooth particle hydrodynamic formulations, Computer Methods in Applied Mechanics and Engineering 180 (1999) 97-115.

[50] J. P. Gray, J. J. Monaghan, R. P. Swift, SPH elastic dynamics, Computer Methods in Applied Mechanics and Engineering 190 (2001) 6641-6662. 
[51] R. Vignjevic, J. Campbell, L. Libersky, A treatment of zero-energy modes in the smoothed particle hydrodynamics method, Computer Methods in Applied Mechanics and Engineering 184 (2000) 67-85.

[52] M. A. Puso, A highly efficient enhanced assumed strain physically stabilized hexahedral element, International Journal for Numerical Methods in Engineering 49 (2000) 10291064 .

[53] P. W. Randles, L. D. Libersky, Boundary conditions for a dual particle method, Computers and Structures 83 (2005) 1476-1486.

[54] J. J. Sanchez, P. W. Randles, Dynamic failure simulation of quasi-brittle material in dual particle dynamics, International Journal for Numerical Methods in Engineering 91 (2012) 1227-1250.

[55] J. J. Sanchez, P. W. Randles, A quasi-static dual particle method for solids based on dual particle dynamics, International Journal for Numerical Methods in Engineering 94 (2013) 183-203.

[56] A. Huerta, S. Fernández-Méndez, Locking in the incompressible limit for the elementfree Galerkin method, International Journal for Numerical Methods in Engineering 51 (2001) 1361-1383.

[57] S. Fernández-Méndez, A. Huerta, Imposing essential boundary conditions in mesh-free methods, Computer Methods in Applied Mechanics and Engineering 193 (2004) 12571275 .

[58] W. Elmer, J. S. Chen, M. Puso, E. Taciroglu, A stable, meshfree, nodal integration method for nearly incompressible solids, Finite Elements in Analysis and Design 51 (2012) 81-85.

[59] T. J. R. Hughes, Generalization of selective integration procedures to anisotropic and nonlinear media, International Journal for Numerical Methods in Engineering 15 (1980) $1413-1418$.

[60] J. Donea, A. Huerta, Finite element methods for flow problems, Wiley and Sons, 2004.

[61] J. C. Simo, N. Tarnow, A new energy and momentum conserving algorithm for the nonlinear dynamics of shells, International Journal for Numerical Methods in Engineering 37 (1994) 2527-2549.

[62] S. K. Lahiri, J. Bonet, J. Peraire, A variationally consistent mesh adaptation method for triangular elements in explicit Lagrangian dynamics, International Journal for Numerical Methods in Engineering 82 (2010) 1073-1113.

[63] J. Feldman, J. Bonet, Dynamic refinement and boundary contact forces in SPH with applications in fluid flow problems, International Journal for Numerical Methods in Engineering 72 (2007) 295-324. 
The highlights of the paper are as follows,

- First, a new SPH computational framework for explicit fast solid dynamics is presented. The proposed methodology explores the use of the Streamline Upwind Petrov Galerkin (SUPG) stabilisation methodology as an alternative to the JamesonSchmidt-Turkel (JST) stabilisation recently presented by the authors.

- Second, the variationally consistent nature of the SUPG stabilisation allows for the introduction of a locally preserving angular momentum procedure which can be solved in a monolithic manner.

- Third, evaluation of expensive harmonic and bi-harmonic operators, necessary for the JST stabilisation, is circumvented in the new SUPG-SPH framework.

- Fourth, the SUPG-SPH framework is more accurate (for the same number of degrees of freedom) than its JST-SPH counterpart.

- Fifth, a series of challenging numerical examples (e.g. in the near incompressibility regime) are examined in order to assess the robustness and accuracy of the proposed algorithm. The obtained results are benchmarked against a wide spectrum of alternative numerical strategies.

Yours faithfully

The authors 